\title{
Complex Dynamics in Generalized Hénon Map
}

\author{
Meixiang Cai \\ Institute of Mathematics and Physics, Central South University of Forestry and Technology, Changsha, Hunan 410004, China
}

Correspondence should be addressed to Meixiang Cai; cmx19811981@sina.com

Received 19 November 2014; Accepted 10 February 2015

Academic Editor: Viktor Avrutin

Copyright (C) 2015 Meixiang Cai. This is an open access article distributed under the Creative Commons Attribution License, which permits unrestricted use, distribution, and reproduction in any medium, provided the original work is properly cited.

\begin{abstract}
The complex dynamics of generalized Hénon map with nonconstant Jacobian determinant are investigated. The conditions of existence for fold bifurcation, flip bifurcation, and Hopf bifurcation are derived by using center manifold theorem and bifurcation theory and checked up by numerical simulations. Chaos in the sense of Marotto's definition is proved by analytical and numerical methods. The numerical simulations show the consistence with the theoretical analysis and reveal some new complex phenomena which can not be given by theoretical analysis, such as the invariant cycles which are irregular closed graphics, the six and forty-one coexisting invariant cycles, and the two, six, seven, nine, ten, and thirteen coexisting chaotic attractors, and some kinds of strange chaotic attractors.
\end{abstract}

\section{Introduction}

The planar map

$$
\begin{gathered}
x_{n+1}=1+a y_{n}-b x_{n}^{2}, \\
y_{n+1}=x_{n}
\end{gathered}
$$

was first introduced by Hénon [1] as a planar diffeomorphism that imitated essential stretching and folding properties of the Poincaré map of the Lorenz system. This original Hénon map (1) had a strange attractor with fractal structure and had constant Jacobian determinant $|J|=-b$. Since the late 1970s, Hénon map (1) served as an important but artificial example to illustrate many analytical results and numerical techniques of dynamical theory. For example, Feit [2] introduced the characteristic exponents in order to estimate strange attractors numerically. Marotto [3] proved analytically that the map had a transversal homoclinic orbit, which implied the existence of the chaotic behavior for some parameter values. Curry [4] presented a lot of numerical experiments on Hénon map by using the characteristic exponent, frequency spectrum, and a theorem of Smale [5]. Mora and Viana [6] proposed the more general theory of strange attractors. Sonis [7] presented a detailed description of bifurcation phenomena by using the analysis approach. Cao [8] proved that there exists a set with positive Lebesgue measure, which corresponded to a map possessing a strange attractor. Luo and Guo [9] investigated the complete bifurcation and stability of the stable and unstable periodic solutions and the chaotic layers by introducing the positive and negative iterative mappings. Zhang [10] verified the existence of Wada basin boundaries in a switched Hénon map.

For the measure preserving Hénon map $(|J|=-b=-1)$, there has been some attention. Such as, Brown [11] proved that the measure preserving Hénon map contained an embedded horseshoe for $a>0$ by using geometric methods and a contradiction argument. Kirchgraber and Stoffer [12] proved this Hénon map existing a transversal homoclinic point for a set of parameters which was not small by using shadowing techniques. Jensen [13] proved that the unstable manifold of a hyperbolic fixed point was the iterated limit of a very simple set.

In this paper, we study the following extension of map (1):

$$
\begin{array}{r}
x_{n+1}=1+a y_{n}-b x_{n}^{2}+c x_{n} y_{n}+d y_{n}^{3}, \\
y_{n+1}=x_{n}+e y_{n}, \\
(c \neq 0,1),
\end{array}
$$

where $b, c$, and $d$ are constants. Following [14], we call this map the generalized Hénon map (GHM), which has nonconstant Jacobian determinant $|J|=-(2 b e+c) x-3 d y^{2}+$ cey $-a$.

Our motivation to study this particular generalized Hénon map is that it appears in the bifurcation analysis of 
nontransversal homoclinic orbits and heteroclinic cycles and plays an important role in other homoclinic studies. In [15], if a diffeomorphism in $R^{2}$ has two saddle fixed points connected by two heteroclinic orbits and one of which is nontransversal, then the GHM appears as a rescaled first return map. In [16], If a diffeomorphism in $R^{3}$ has a codimension 1 homoclinic tangency to a saddle-focus fixed point, the GHM appears when the eigenvalues satisfy some conditions. Moreover, when the so-called effective dimension of the problem in [17] can change, the GHM also can be expected as a rescaled first-return map in other cases of homoclinic and heteroclinic tangencies.

In this paper, the bifurcations and chaos phenomenons in map (2) are investigated. The conditions of existence for fold bifurcation, flip bifurcation, and Hopf bifurcation are derived by using center manifold theorem and bifurcation theory [18]; chaotic behavior in the sense of Marotto's definition [19] is proved. And numerical simulation results not only show the consistence with the theoretical analysis but also display the new and interesting dynamics behaviors.

This paper is organized as follows. In Section 2, the existence and stability of the fixed points are given. In Section 3 , the sufficient conditions of existence for fold bifurcation, flip bifurcation, and Hopf bifurcation are given. In Section 4, we first rigorously prove the existence of chaos in the sense of Marotto's definition and then give an example to check up the analytic results. The numerical simulations are presented in Section 5 to verify the theoretical analysis and display the new and interesting dynamics. Finally, we give a conclusion in Section 6.

\section{Existence and Stability of Fixed Points}

The fixed points of map (2) satisfy the following equations:

$$
\begin{gathered}
1+a y-b x^{2}+c x y+d y^{3}=x, \\
x+e y=y .
\end{gathered}
$$

By a simple analysis about (3), it is easy to obtain the following proposition.

\section{Proposition 1.}

(i) If $\Delta>0$, map (2) has a unique fixed point at $Z_{1}\left(x_{1}^{0}, y_{1}^{0}\right)$,

(ii) if $\Delta=0$, map (2) has two fixed points at $Z_{1}\left(x_{1}^{0}, y_{1}^{0}\right)$ and $Z_{2}\left(x_{2}^{0}, y_{2}^{0}\right)$,

(iii) if $\Delta<0$, map (2) has three fixed points at $Z_{1}\left(x_{1}^{0}, y_{1}^{0}\right)$, $Z_{2}\left(x_{2}^{0}, y_{2}^{0}\right)$, and $Z_{3}\left(x_{3}^{0}, y_{3}^{0}\right)$,

where $\Delta=4\left[3 d(a+e-1)-(1-e)^{2}(c-b+b e)^{2}\right]^{3}+[2(1-$ $\left.e)^{3}(c-b+b e)^{3}-9 d(1-e)(c-b+b e)(a+e-1)+27 d^{2}\right]^{2}$.

The Jacobian matrix $J$ of map (2) evaluated at fixed point $Z(x, y)$ is given by

$$
J=\left(\begin{array}{cc}
-2 b x+c y & a+c x+3 d y^{2} \\
1 & e
\end{array}\right) .
$$

The characteristic equation of the Jacobian matrix $J$ can be written as

$$
\lambda^{2}+p(x, y) \lambda+q(x, y)=0
$$

where $p(x, y)=2 b x-c y-e$ and $q(x, y)=-(2 b e+c) x-$ $3 d y^{2}+c e y-a$.

A simple calculation shows the stability of the fixed points as the following proposition.

Proposition 2. The fixed point $Z(x, y)$ of map (2) is stable if one of the following conditions holds:

$$
\begin{aligned}
& \text { (i) }-1<-(2 b e+c) x-3 d y^{2}+c e y-a<1 \text {; } \\
& \text { (ii) }-(2 b e+c) x-3 d y^{2}+c e y-a<1 \text { and }(2 b-2 b e+c) x- \\
& 3 d y^{2}+c(e-1) y+1-e-a>0 \text { and }-(2 b+2 b e+c) x- \\
& 3 d y^{2}+c(e+1) y+1+e-a>0 .
\end{aligned}
$$

\section{Bifurcations}

In analysis of the fold bifurcation, flip bifurcation, and Hopf bifurcation of map (2), $b$ is used as the bifurcation parameter. By using center manifold theorems and bifurcation theory, the conditions of existences for the bifurcations are given as follows.

Theorem 3. Map (2) undergoes a fold bifurcation at $Z\left(x\left(b_{0}\right)\right.$, $\left.y\left(b_{0}\right)\right)$ if the following conditions are satisfied at the same time:

$$
\begin{gathered}
b_{0} \neq \frac{c y_{0}+e}{2 x_{0}}, \quad b_{0} \neq \frac{c y_{0}+e-2}{2 x_{0}}, \\
b_{0}=\frac{c x_{0}+3 d y_{0}^{2}+c(1-e) y_{0}+a+e-1}{2(1-e) x_{0}}, \\
\alpha=\frac{1}{A^{2}}\left[b_{0}(1-e)^{2}-c(1-e)-3 d y_{0}\right] \neq 0 .
\end{gathered}
$$

Moreover, if $\alpha<0$ (resp., $\alpha>0$ ), the two fixed points bifurcate from $Z$ for $b>b_{0}$ (resp., $b<b_{0}$ ) and coalesce as the fixed point $Z$ at $b=b_{0}$ and disappear for $b<b_{0}$ (resp., $b>b_{0}$ ) (see Appendix A for the proof).

Theorem 4. Map (2) undergoes a flip bifurcation at $Z\left(x\left(b^{*}\right)\right.$, $\left.y\left(b^{*}\right)\right)$ if the following conditions are satisfied at the same time:

$$
\begin{gathered}
b^{*} \neq \frac{c y_{0}+e+1}{2 x_{0}}, \quad b^{*} \neq \frac{c y_{0}+e+3}{2 x_{0}}, \\
b^{*} \neq \frac{-c x_{0}-3 d y_{0}^{2}+c e y_{0}-a}{2 e x_{0}}, \\
b^{*}=\frac{-c x_{0}-3 d y_{0}^{2}+c(1+e) y_{0}-a+e+1}{2(1+e) x_{0}}, \\
\alpha_{1} \neq 0, \quad \alpha_{2} \neq 0 .
\end{gathered}
$$

Moreover, if $\alpha_{2}>0$ (resp., $\alpha_{2}<0$ ), the period-2 points that bifurcate from this fixed point are stable (resp., unstable) (see Appendix $B$ for the proof and expressions of $\alpha_{1}$ and $\alpha_{2}$ ).

Theorem 5. Map (2) undergoes a Hopf bifurcation at $Z(x(\bar{b})$, $y(\bar{b}))$ if the following conditions are satisfied at the same time:

$$
\begin{gathered}
\Delta^{\prime}=-x_{0}^{2}\left(3 d y_{0}^{2}+c x_{0}+a\right)>0, \\
\frac{-x_{0}\left(e-c y_{0}\right)-2 \sqrt{\Delta^{\prime}}}{2 x_{0}^{2}}<b<\frac{-x_{0}\left(e-c y_{0}\right)+2 \sqrt{\Delta^{\prime}}}{2 x_{0}^{2}}
\end{gathered}
$$




$$
\begin{gathered}
\bar{b} \neq \frac{c y_{0}+e}{2 a x_{0}}, \quad \bar{b} \neq \frac{c y_{0}+e+1}{2 a x_{0}}, \\
\bar{b}=-\frac{3 d y_{0}^{2}+c\left(x_{0}-e y_{0}\right)+a+1}{2 e x_{0}}, \quad l \neq 0 .
\end{gathered}
$$

Moreover, if $l<0($ resp., $l>0)$ and $k=\left.(d|\lambda(b)| / d b)\right|_{b=\bar{b}}>$ 0 ; then, an attracting (resp., repelling) invariant closed curve bifurcates from the fixed point for $b>\bar{b}$ (resp., $b<\bar{b}$ ) (see Appendix $C$ for the proof and expression of $l$ ).

\section{Existence of Marotto's Chaos}

In this section, we rigorously prove that map (2) possesses chaotic behavior in the sense of Marotto's definition in [19].

Suppose that $Z_{0}\left(x_{0}, y_{0}\right)$ is the fixed point of map (2). We first give the conditions such that the fixed point $Z_{0}$ is a snapback repeller. The eigenvalues associated with the fixed point $Z_{0}$ are given by

$$
\lambda_{1,2}=\frac{-p\left(y_{0}\right) \pm \sqrt{p^{2}\left(y_{0}\right)-4 q\left(y_{0}\right)}}{2},
$$

where $p\left(y_{0}\right)=[2 b(1-e)-c] y_{0}-e$ and $q\left(y_{0}\right)=-3 d y_{0}^{2}-$ $[2 b e(1-e)+c(1-2 e)] y_{0}-a$.

According to Definition 1 in [19], we begin to find a set $U_{Z_{0}}$ in which the norm of conjugate complex eigenvalues exceeds 1 for all $Z \in U_{Z_{0}}$. Let $H(\lambda)=\lambda^{2}+p(y) \lambda+q(y)=0$, which represents the characteristic equation for any point $(x, y)$ in $\mathbb{R}^{2}$. If $\Delta=p^{2}(y)-4 q(y)<0$ and $q(y)>1$, then the characteristic equation have a pair of conjugate complex eigenvalues satisfying $\left|\lambda_{1,2}\right|>1$.

Let

$$
\begin{aligned}
\varphi_{1}(y)=\Delta= & {\left[(2 b-2 b e-c)^{2}+12 d\right] y^{2} } \\
& +2\left(2 b e-2 b e^{2}-3 c e+2 c\right) y+4 a+e^{2}, \\
\varphi_{2}(y)= & q(y)-1 \\
= & -3 d y^{2}-[2 b e(1-e)+c(1-2 e)] y-a-1 ;
\end{aligned}
$$

then,

$$
\begin{aligned}
& \Delta_{1}^{\prime}=16\{[c(4-b e)-4 b e(e-1)]^{2} \\
&- 4\left[c^{2}+12 d+4 b c(e-1)+4 b^{2}(e-1)^{2}\right] \\
&\left.\cdot\left(4 a+e^{2}\right)\right\}, \\
& \Delta_{2}^{\prime}=[2 b e(1-e)+c(1-2 e)]^{2}-12 d(a+1) .
\end{aligned}
$$

If $\Delta_{1}^{\prime}>0$, the roots of $\varphi_{1}(y)=0$ are $y_{1,2}=\left(-2 b e+2 b e^{2}+\right.$ $\left.3 c e-2 c \pm 2 \sqrt{\Delta_{1}^{\prime}}\right) /\left((2 b-2 b e-c)^{2}+12 d\right)$, and if $\Delta_{2}^{\prime}>0$, the roots of $\varphi_{2}(y)=0$ are $y_{3,4}=\left(2 b e(1-e)+c(1-2 e) \pm \sqrt{\Delta_{2}^{\prime}}\right) / 6 d$.

From the above discussion, we obtain the following results.
Lemma 6. Supposing that $D_{1}=\left\{y \mid p^{2}(y)-4 q(y)<0\right\}$, $D_{2}=\{y \mid q(y)>1\}$ and $D=D_{1} \cap D_{2}$, we have the following.

(i) If $(2 b-2 b e-c)^{2}+12 d>0, d>0, \Delta_{1}^{\prime}>0, \Delta_{2}^{\prime}>0$, and $y_{1} \leq y_{3}<y_{2}$ or $y_{1}<y_{4} \leq y_{2}$ or $y_{3} \leq y_{1}<y_{4}$ or $y_{3}<y_{2} \leq y_{4}$, then $D=\left(y_{1}, y_{2}\right) \cap\left(y_{3}, y_{4}\right) \neq \emptyset$.

(ii) If $(2 b-2 b e-c)^{2}+12 d>0, d<0, \Delta_{1}^{\prime}>0, \Delta_{2}^{\prime}>0$, and $y_{1}<y_{3}$ or $y_{2}>y_{4}$, then $D=\left(y_{1}, y_{2}\right) \cap\left(\left(-\infty, y_{3}\right) \cup\right.$ $\left.\left(y_{4},+\infty\right)\right) \neq \emptyset$.

(iii) If $(2 b-2 b e-c)^{2}+12 d>0, d<0, \Delta_{1}^{\prime}>0, \Delta_{2}^{\prime}<0$, then $D=\left(y_{1}, y_{2}\right) \neq \emptyset$.

(iv) If $(2 b-2 b e-c)^{2}+12 d<0, d<0, \Delta_{1}^{\prime}>0, \Delta_{2}^{\prime}>0$, then $D=\left(\left(-\infty, y_{1}\right) \cup\left(y_{2},+\infty\right)\right) \cap\left(\left(-\infty, y_{3}\right) \cup\left(y_{4},+\infty\right)\right) \neq$ $\emptyset$.

(v) If $(2 b-2 b e-c)^{2}+12 d<0, d<0, \Delta_{1}^{\prime}>0, \Delta_{2}^{\prime}<0$, then $D=\left(-\infty, y_{1}\right) \cup\left(y_{2},+\infty\right) \neq \emptyset$.

(vi) If $(2 b-2 b e-c)^{2}+12 d<0, d<0, \Delta_{1}^{\prime}<0, \Delta_{2}^{\prime}>0$, then $D=\left(-\infty, y_{3}\right) \cup\left(y_{4},+\infty\right) \neq \emptyset$.

(vii) If $(2 b-2 b e-c)^{2}+12 d<0, d<0, \Delta_{1}^{\prime}<0, \Delta_{2}^{\prime}<0$, then $y$ is arbitrary.

Lemma 7. If one of the conditions in Lemma 6 is satisfied and the $y$-coordinate of fixed point $Z_{0}\left(x_{0}, y_{0}\right)$ satisfies $y_{0} \in D_{y_{0}} \subset$ $D$, then $Z_{0}\left(x_{0}, y_{0}\right)$ is an expanding fixed point of map (2) in $U_{Z_{0}}=\left\{(x, y) \mid y \in D_{y_{0}}, x \in \mathbb{R}\right\}$.

Due to Definition 2 of snap-back repeller in [19], we need to find one point $Z \in U_{Z_{0}}$ such that $Z \neq Z_{0}, F^{M}(Z)=Z_{0}$, and $\left|D F^{M}(Z)\right| \neq 0$ for some positive integer $M$.

In fact, we have

$$
\begin{gathered}
1-a y-b x^{2}+c x y+d y^{3}=x_{1}, \\
x+e y=y_{1}, \\
1-a y_{1}-b x_{1}^{2}+c x_{1} y_{1}+d y_{1}^{3}=x_{0}, \\
x_{1}+e y_{1}=y_{0} .
\end{gathered}
$$

Now, a map $F^{2}$ has been constructed to map the point $Z(x, y)$ to the fixed point $Z_{0}\left(x_{0}, y_{0}\right)$ after two iteration if there are solutions different from $Z_{0}$ for (12) and (13). By calculation, the solutions different from $Z_{0}$ for (13) are

$$
\begin{aligned}
y_{1}^{ \pm}=( & -d y_{0}+b e^{2}+c e \\
\pm( & -3 d^{2} y_{0}^{2}+2\left(b d e^{2}+c d e-2 c d-4 b d e\right) y_{0} \\
& \left.\left.+b^{2} e^{4}+2 b c e^{3}+c^{2} e^{2}-4 a d\right)^{1 / 2}\right)(2 d)^{-1}, \\
x_{1}^{ \pm}=y_{0}-e y_{1}^{ \pm} . &
\end{aligned}
$$

Substituting (14) into (12), we have

$$
\begin{aligned}
& d y^{3}-\left(b e^{2}+c e\right) y^{2}+\left(a+2 b e y_{1}+c y_{1}\right) y-b y_{1}^{2} \\
& +e y_{1}-y_{0}+1=0,
\end{aligned}
$$

where $y_{1}=y_{1}^{ \pm}$. 


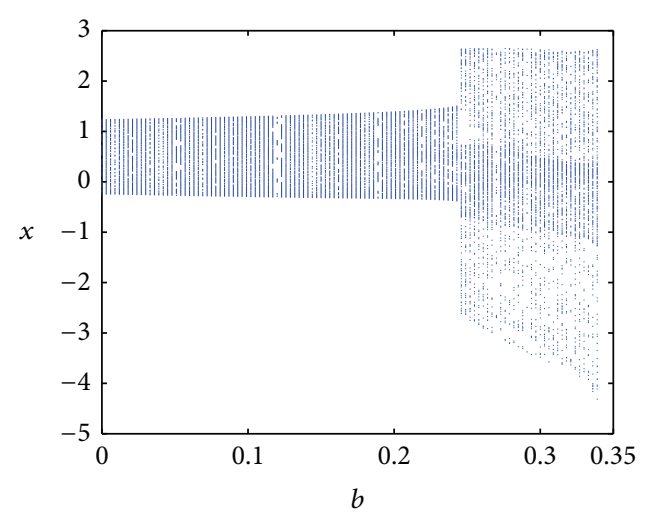

(a)

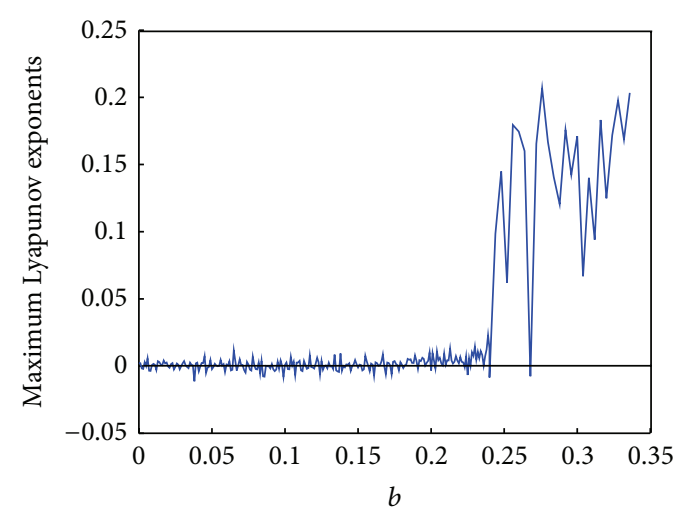

(b)

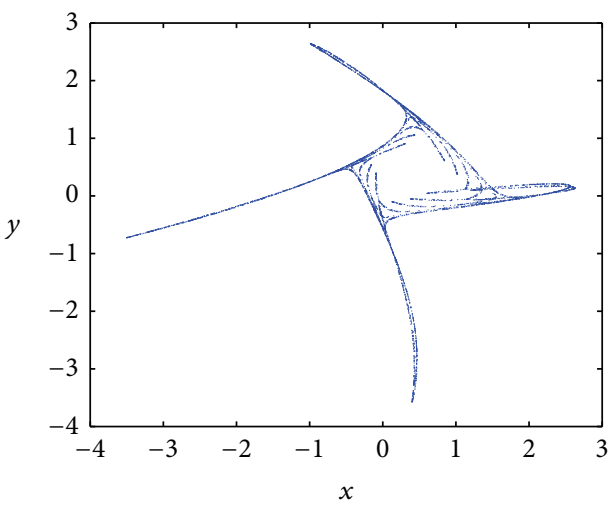

(c)

Figure 1: (a) Bifurcation diagram of map (2) in $(b, x)$ plane for $a=-1.5, c=0.8, d=0.1$, and $e=0.1$. (b) Maximum Lyapunov exponents corresponding to (a). (c) Phase portrait of the chaotic attractor for $a=-1.5, b=0.3, c=0.8, d=0.1$, and $e=0.1$.

Next, we expect to find a real root of (15) satisfying $y^{*} \epsilon$ $D_{y_{0}}$. Let $y=u+y_{0}$, then (15) becomes

$$
\begin{aligned}
& d u^{3}+\left(3 d y_{0}-b e^{2}-c e\right) u^{2} \\
& +\left(3 d y_{0}^{2}-2 b e^{2} y_{0}-2 c e y_{0}+a+c y_{1}+2 b e y_{1}\right) u \\
& +d y_{0}^{3}-\left(b e^{2}+c e\right) y_{0}^{2}+(a-1) y_{0}+1+e y_{1} \\
& +c y_{0} y_{1}+2 b e y_{0} y_{1}-b y_{1}^{2}=0
\end{aligned}
$$

Let $u=v-\left(3 d y_{0}-b e^{2}-c e\right) / 3 d$; then, (16) becomes

$$
v^{3}+P v+Q=0
$$

where $P=\left(1 / 3 d^{2}\right)\left(3 a d-c^{2} e^{2}-2 b c e^{3}-b^{2} e^{4}+3 c d y_{1}+6 b d e y_{1}\right)$, $Q=\left(1 / 27 d^{3}\right)\left\{-2 e^{3}(c+b e)^{3}+9 d e(c+b e)\left[a+(c+2 b e) y_{1}\right]-\right.$ $\left.27 d^{2}\left(-1+y_{0}-e y_{1}+b y_{1}^{2}\right)\right\}$, and $\Delta=(P / 3)^{3}+(Q / 2)^{2}$.

If the real root of (17) $v^{*} \neq\left(3 d y_{0}-b e^{2}-c e\right) / 3 d$, then (15) has at least a real root $y^{*} \neq y_{0}$.

Obviously, if one of the conditions in Lemma 6 is satisfied and $v^{*} \neq\left(3 d y_{0}-b e^{2}-c e\right) / 3 d$ and the solution of (15) satisfies $y^{*} \in U_{Z_{0}}$, then $Z_{0}$ is a snap-back repeller in $U_{Z_{0}}$. Thus, we have the following theorem.
Theorem 8. If one of the conditions in Lemma 6 holds and $v^{*} \neq\left(3 d y_{0}-b e^{2}-c e\right) / 3 d$ and the solutions $\left(x_{1}^{ \pm}, y_{1}^{ \pm}\right)$and $\left(x^{*}, y^{*}\right)$ of (14) and (15) satisfy $y_{1}^{ \pm}, y^{*} \neq y_{0},\left(x^{*}, y^{*}\right) \in U_{Z_{0}}$, then the point $Z_{0}\left(x_{0}, y_{0}\right)$ is a snap-back repeller of map (2), and hence, map (2) is chaotic in the sense of Marotto's definition, where $U_{Z_{0}}$ is given in Lemma 7.

Next, we give specific values of the parameters for illustrating and verifying the conditions in Theorem 8 .

Example 9. For $a=-1.5, b=0.3, c=0.8, d=0.1, e=0.1$, map (2) has a fixed point $Z_{0}\left(x_{0}, y_{0}\right)=(0.417165,0.463517)$, and the eigenvalues of the fixed point $Z_{0}$ are $\lambda_{+}=0.110257 \pm$ 1.19306i. From Lemma 6, we have $D_{1}=(-41.6035,39.3725)$, $D_{2}=(-0.576696,2.89003)$, and $D=(-0.576696,2.89003)$, which shows that $y_{0}=0.463517 \in D_{y_{0}} \subset D$. We choose $y_{1}=y_{1}^{+}=3.53245 \notin D$; then, the solution of (15) is $y^{*}=1.63427 \neq y_{0}$ and $y^{*} \in D_{y_{0}}$. There exists a point $Z\left(x^{*}\right.$, $\left.y^{*}\right)=(3.36302,1.69427) \in U_{Z_{0}}$, which is different from $Z_{0}$ and satisfies $F^{2}(Z)=Z_{0}$ and $D F^{2}(Z) \neq 0$. So; $Z_{0}$ is a snapback repeller.

The bifurcation diagram and the corresponding maximum Lyapunov exponents of map $(2)$ in $(b, x)$ plane for $a=$ $-1.5, c=0.8, d=0.1$, and $e=0.1$ are shown in Figures $1(\mathrm{a})$ and 1(b), respectively. The attractor of map (2) for $a=-1.5$, 


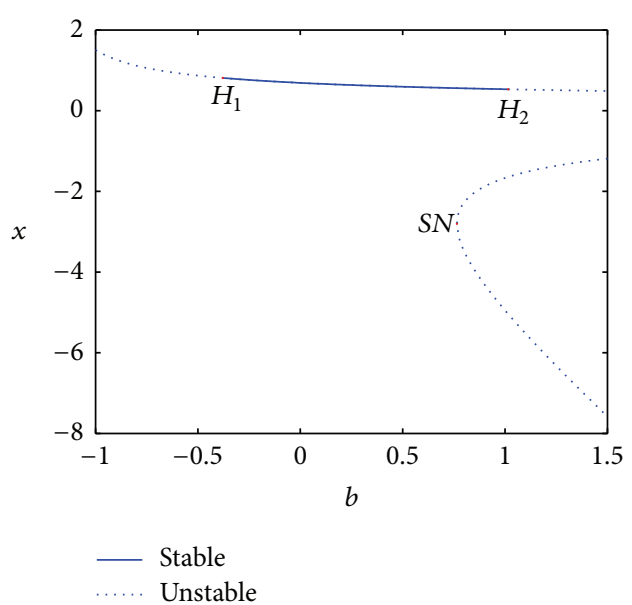

(a)

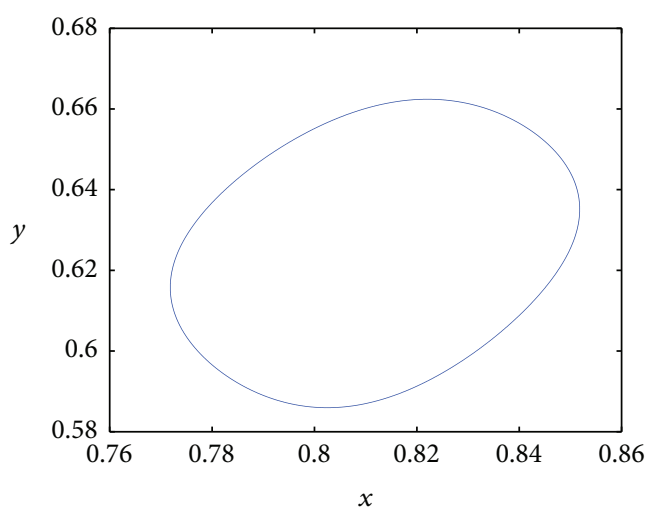

(b)

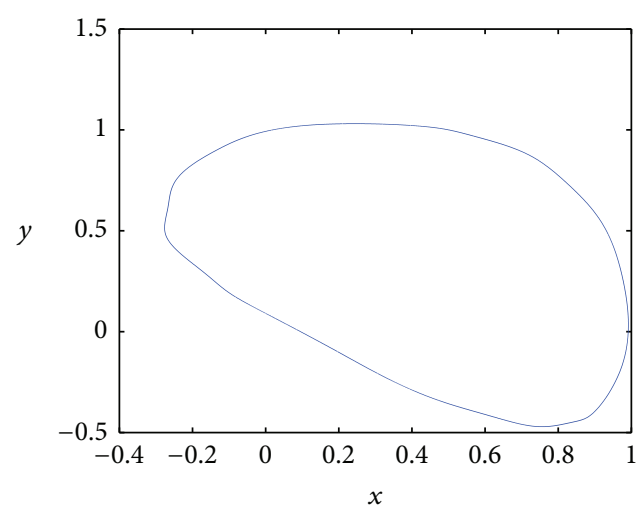

(c)

Figure 2: (a) Bifurcation diagram of the fixed points of map (2) in $(b, x)$ plane for $a=-0.1, c=-0.5, d=-0.5$, and $e=-0.3$; (b) and (c): invariant cycles corresponding to Figure $2(\mathrm{a})$ at $b=-0.38$ and $b=1.02$, respectively.

$b=0.3, c=0.8, d=0.1$, and $e=0.1$ is given in Figure $1(\mathrm{c})$ (where the maximum Lyapunov exponent $L=0.168>0$ ), which is a Maratto's chaotic attractor.

\section{Numerical Simulations}

In this section, we use the bifurcation diagrams, Lyapunov exponents, and phase portraits to illustrate the above analytic results and show the new and more complex dynamical behaviors in the map (2).

5.1. Numerical Simulations for Stability and Codimension One Bifurcations of Fixed Points. In this sbusection, we give the numerical simulations for verifying Propositions 1 and 2 and Theorems 3-5. Four cases are considered as the follows.

Case 1. The bifurcation diagram of fixed points of map (2) in $(b, x)$ plane for $a=0.7, c=0.3, d=-0.15$, and $e=0.08$ is given in Figure 2(a). We show that map (2) has one, two, and three fixed points for $b<0.764782, b=$ 0.764782 , and $b>0.764782$, respectively. The fold bifurcation (labeled "SN") occurs at the fixed point $(-2.80455,-2.15734)$ for $b_{S N}=0.764782$, and two unstable fixed points bifurcate from this point for $b>b_{S N}$, which check the correctness of Theorem 3 (here the coefficient $\alpha=-0.137389<0$ ). The Hopf bifurcations (labeled " $H_{1}$ " and " $H_{2}$ ") occur at fixed points $(0.811635,0.624334)$ and $(0.530768,0.408283)$ for $b_{H_{1}}=-0.378162$ and $b_{H_{2}}=1.0153$, respectively. For example, an attracting invariant cycle bifurcates from the fixed point $(0.530768,0.408283)$ for $b>b_{H_{2}}$, where the coefficients in Theorem 5 are $l_{\mathrm{H}_{2}}=-2.02282<0$ and $k_{\mathrm{H}_{2}}=$ $0.15923>0$, which check the correctness of Theorem 5 . The stable invariant cycles for $b=-0.38$ and $b=1.02$ are shown in Figures 2(b) and 2(c), respectively.

Case 2. The bifurcation diagram of fixed point of map (2) in $(b, x)$ plane for $a=0.7, c=0.3, d=-0.15$, and $e=0.08$ is given in Figure 3(a). We show that there is only one fixed point for $0 \leq b \leq 0.6$, and the Hopf bifurcation (labeled " $H$ ") occurs at fixed point $(2.07188,2.25205)$ for $b_{H}=0.0445132$, and flip bifurcation (labeled " $P$ - $D$ ") emerges at the fixed point $(1.30361,1.41697)$ for $b_{P-D}=0.479978$, which check the correctness of Theorems 5 and 4 , respectively. In fact, the fixed point is stable for $b_{H}<b<b_{P-D}$ and loses its stability at flip bifurcation value $b=b_{P-D}$, an unstable period-2 points bifurcate from the fixed point as $b$ increasing (here 


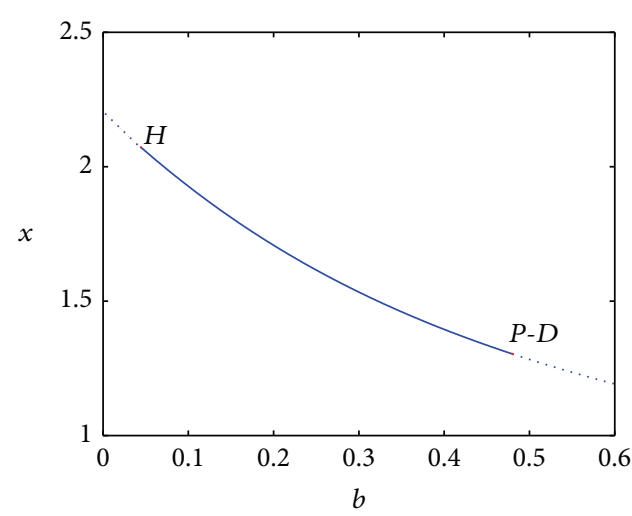

(a)

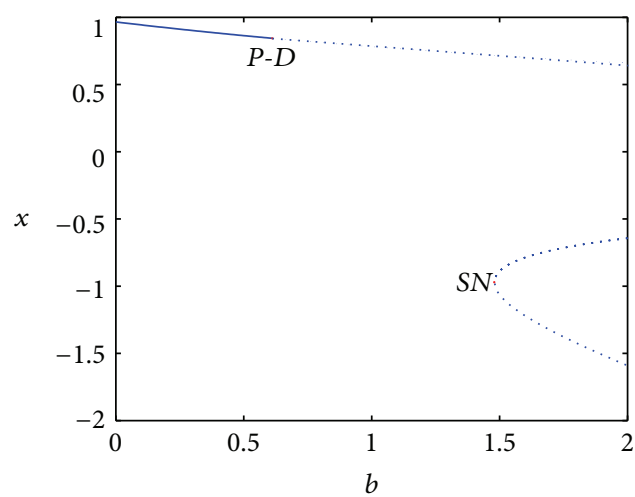

(b)

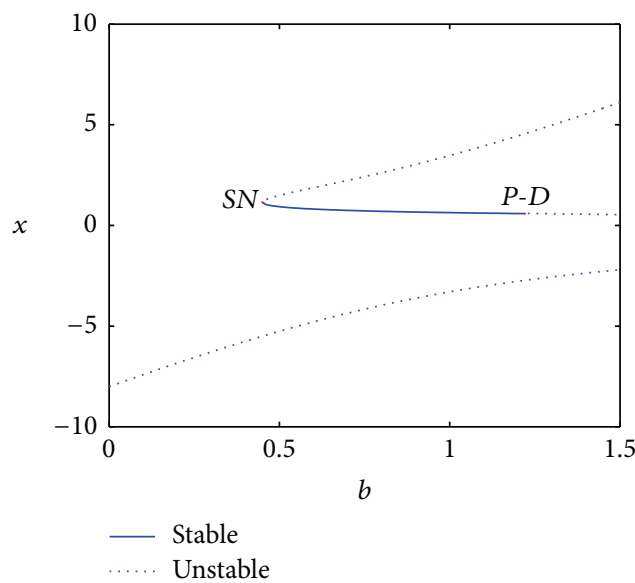

(c)

FIGURE 3: Bifurcation diagrams of the fixed points of map (2) in ( $b, x)$ plane for (a) $a=0.7, c=0.3, d=-0.15$, and $e=0.08$; (b) $a=0.5$, $c=-0.1, d=-0.04$, and $e=0.7$; (c) $a=-0.5, c=0.8, d=0.1$, and $e=0.1$.

the coefficients in Theorem 5 are $\alpha_{1}=16.8788 \neq 0$ and $\left.\alpha_{2}=-0.179751<0\right)$.

Case 3. The bifurcation diagram of fixed point of map (2) in $(b, x)$ plane for $a=0.5, c=-0.1, d=-0.04$, and $e=$ 0.7 is given in Figure 3(b). We show that the flip bifurcation (labeled " $P-D$ ") occurs at fixed point $(0.843666,2.81222)$ for $b_{P-D}=0.611937$, and fold bifurcation (labeled " $S N$ ") emerges from fixed point $(-0.970253,-3.23418)$ for $b_{S N}=1.47923$.

Case 4. The bifurcation diagram of fixed point of map (2) in $(b, x)$ plane for $a=-0.5, c=0.8, d=0.1$, and $e=0.1$ is given in Figure 3(c). We show that the fold bifurcation (labeled " $S N$ ") emerges from fixed point $(1.15118,1.27909)$ for $b_{S N}=$ 0.450125 , and flip bifurcation (labeled " $P-D$ ") occurs at fixed point $(0.587212,0.652457)$ for $b_{P-D}=1.22047$.

5.2. Further Numerical Simulations for Map (2). In this subsection, we study the complex dynamics changes as the parameters varying with the initial value $(0,0)$. The bifurcation parameters are considered in the following five cases.

(a) $0 \leq a \leq 1.3$, and $b=0.02, c=0.3, d=-0.15, e=0.08$.

(b) $0 \leq b \leq 1$, and $a=0.5, c=-0.1, d=-0.04, e=0.7$. (c) $0 \leq c \leq 1$, and $a=0.2, b=1, d=-1.5, e=0.23$.

(d) $-1 \leq d \leq 0.4$, and $a=-0.3, b=0.9, c=-0.8, e=0.5$.

(e) $0.05 \leq e \leq 1.05$, and $a=-0.4, b=1.1, c=-0.6$, $d=-0.55$.

For Case (a). The bifurcation diagram of map (2) in $(a, x)$ plane and the corresponding maximum Lyapunov exponents are shown in Figures 4(a) and 4(b), respectively. Figures 4(c)4(e) are the local amplifications of (a). We observe that there is a stable fixed point for $0 \leq a<0.624$; the fixed point loses its stability as $a$ increases; Hopf bifurcation occurs at $a \sim 0.624$, and the stable invariant cycle at $a=0.625$ is shown in Figure 5(a). The region $a \in(0.624,0.848)$ is the invariant cycle region with complex periodic windows, such as period-16, 27 orbits and the invariant cycles at $a=$ 0.8 is shown in Figure 5(b). The region $a \in(0.848,0.888)$ is the chaotic region with complex periodic windows, such as period-38 orbit (see Figure 5(c)) and period-11 orbits, and the symmetry breaking of period orbits occurs for the region $a \in(0.8535,0.8557)$, and the chaotic attractors at $a=0.862$ and $a=0.877$ are shown in the Figures 5(d) and 5(e), respectively. For $a \sim 0.888$, the chaotic behavior suddenly converges to period -5 orbits, and the period -5 orbits undergo Hopf bifurcations at $a \sim 0.8906$ separately, and 


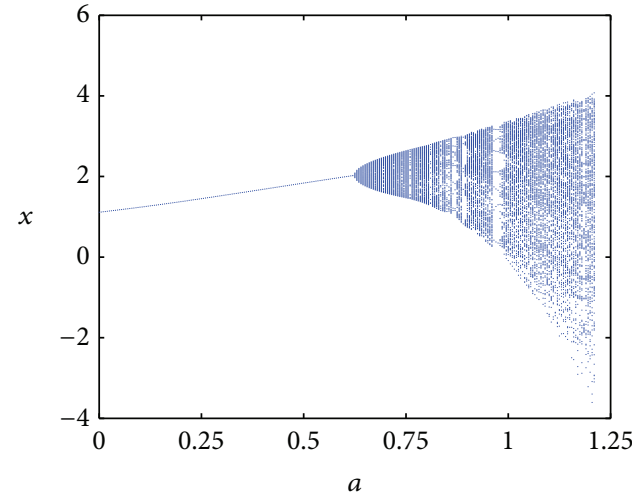

(a)

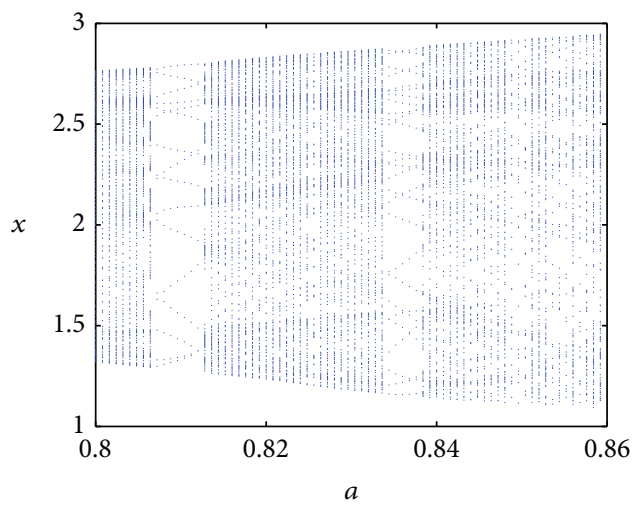

(c)

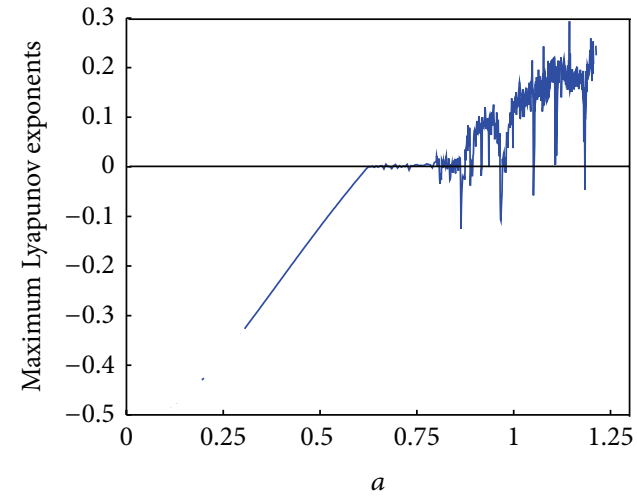

(b)

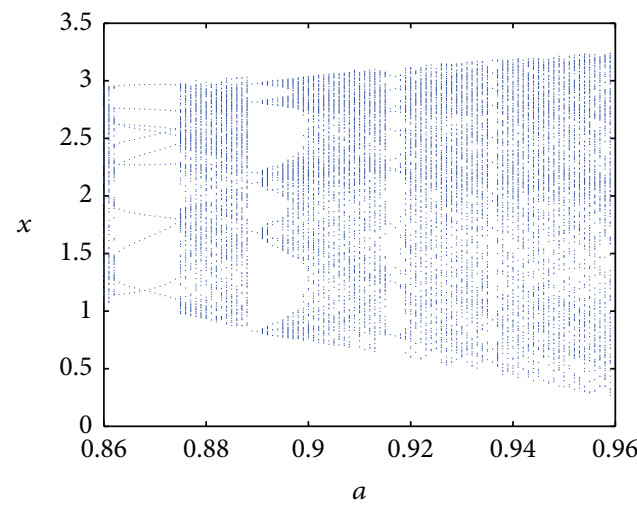

(d)

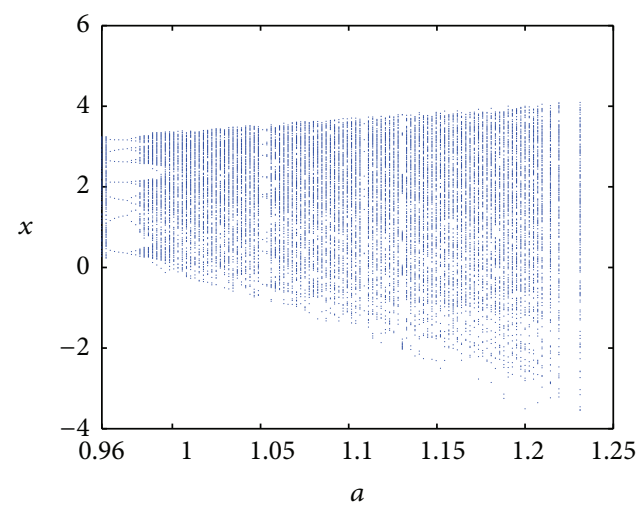

(e)

Figure 4: (a) Bifurcation diagram of map (2) in $(a, x)$ plane for $b=0.02, c=0.3, d=-0.15$, and $e=0.08$. (b) Maximum Lyapunov exponents corresponding to (a). (c)-(e). Local amplification of (a) for (c) $0.8 \leq a \leq 0.86$, (d) $0.86 \leq a \leq 0.96$, and (e) $0.96 \leq a \leq 1.25$.

the six coexisting invariant cycles at $a=0.892$ are shown in Figure 5(f). For the region $a \in(0.8925,1.225)$, we can see the period-doubling bifurcation to chaos and the chaotic behavior suddenly disappears at $a \sim 1.225$, and the six coexisting chaotic attractors at $a=0.895$, the seven coexisting chaotic attractor at $a=0.987$, and the chaotic attractor at $a=1.2$ are shown in the Figures $5(\mathrm{~g})-5(\mathrm{i})$, respectively.

For Case (b). The bifurcation diagram of map (2) in $(b, x)$ plane and the corresponding maximum Lyapunov exponents are shown in Figures 6(a) and 6(b), respectively. In Figure 6, we observe period-doubling bifurcation to chaos and chaotic region with period- 6 windows. The two coexisting chaotic attractors at $b=0.05$ and the chaotic attractor at $b=1$ are shown in Figures 7(a) and 7(c), respectively, and the period-8 orbits at $b=0.85$ is shown in Figure 7(b).

For Case (c). The bifurcation diagram of map (2) in $(c, x)$ plane and the corresponding maximum Lyapunov exponents are shown in Figures 8(a) and 8(b), respectively, which show the chaotic region with complex periodic windows. The chaotic attractors at $c=0.01, c=0.2$, and $c=0.45$ are shown in Figures 9(a)-9(c), respectively. 


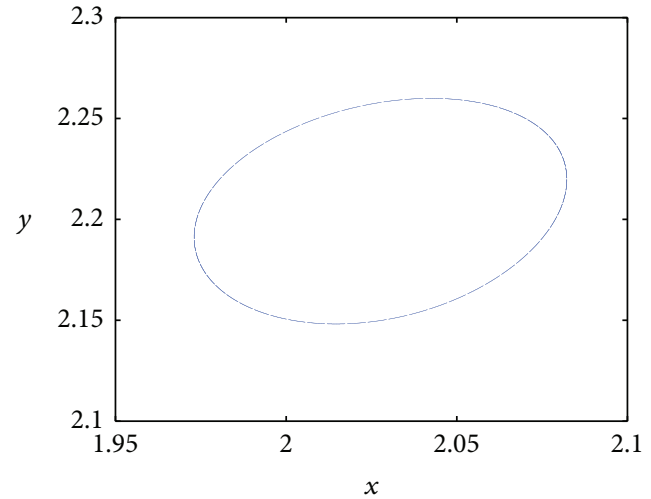

(a)

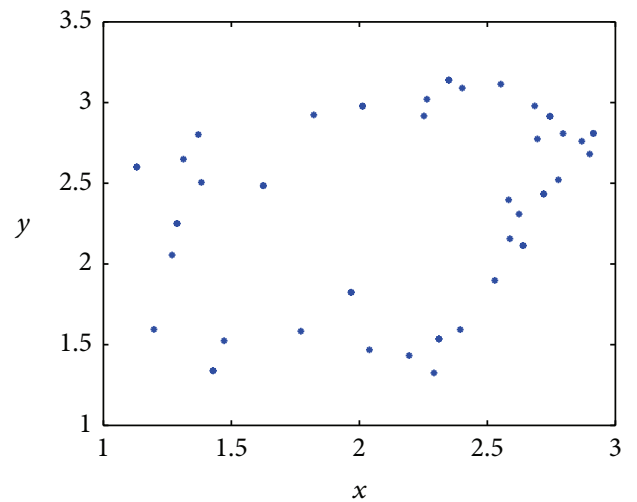

(c)

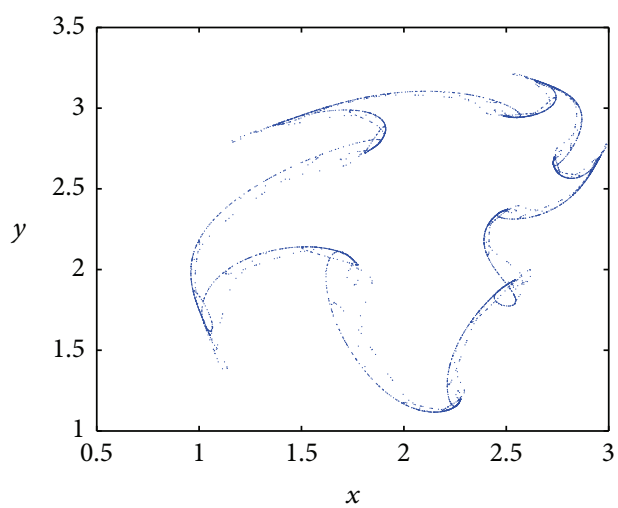

(e)

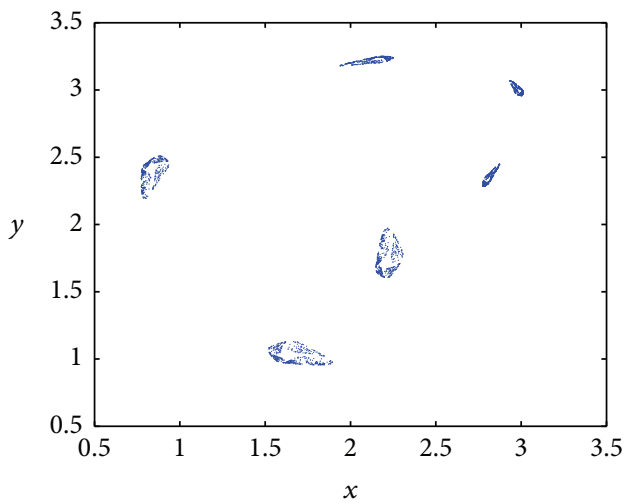

(g)

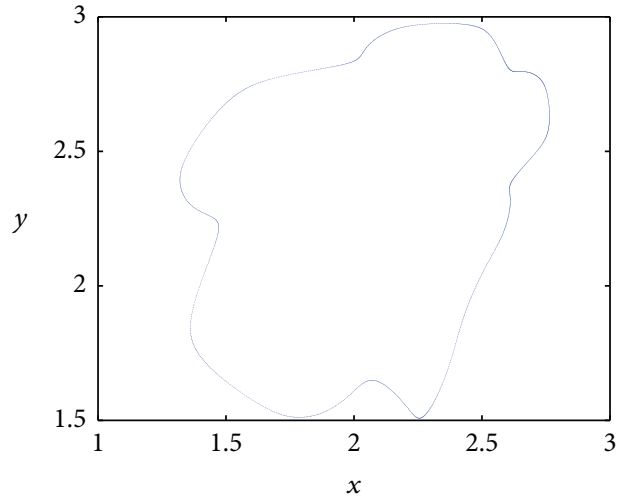

(b)

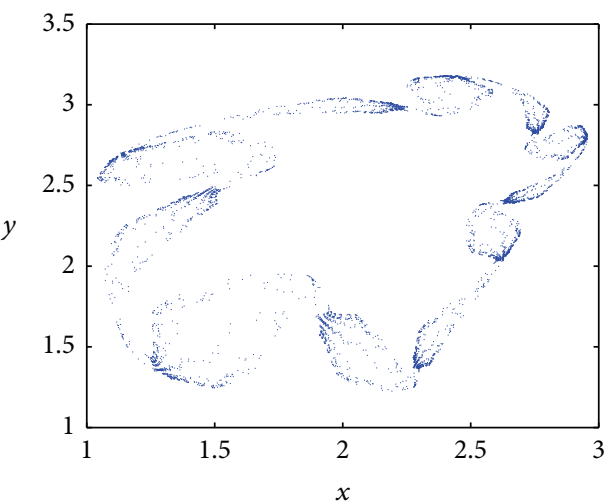

(d)

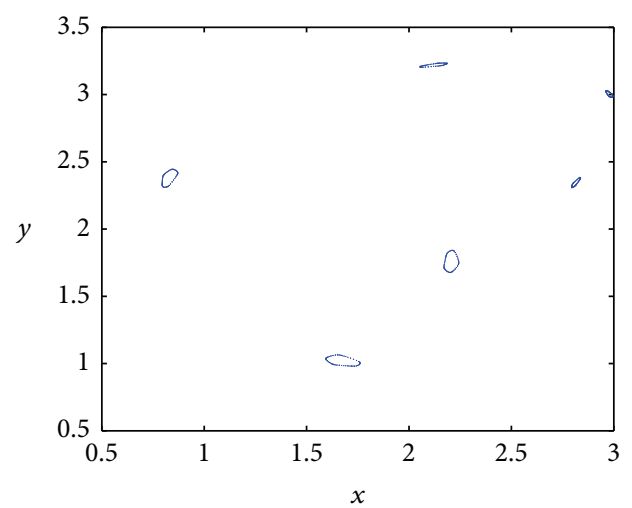

(f)

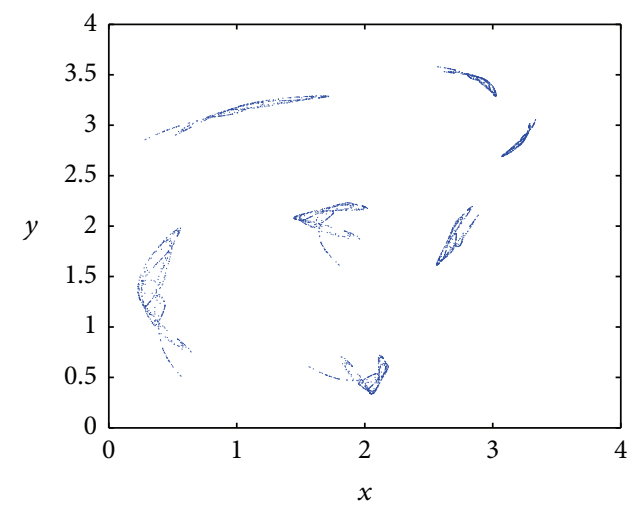

(h)

Figure 5: Continued. 


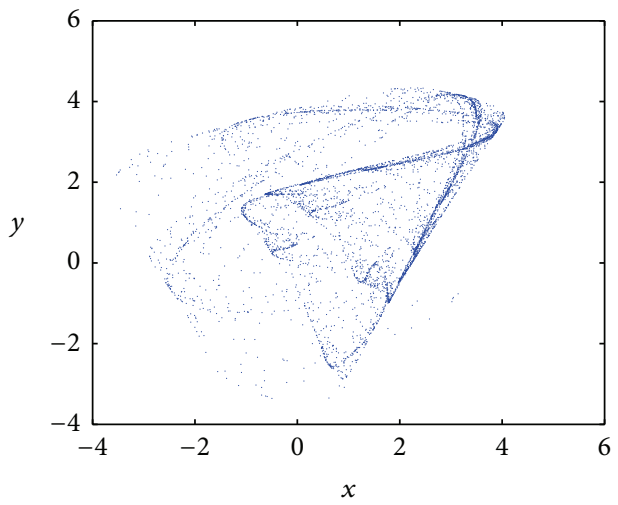

(i)

FIGURE 5: Phase portraits corresponding to Figure 4: (a) $a=0.625$, (b) $a=0.8$, (c) $a=0.85$, (d) $a=0.862$, (e) $a=0.877$, (f) $a=0.892$, (g) $a=0.895$, (h) $a=0.987$, and (i) $a=1.2$.

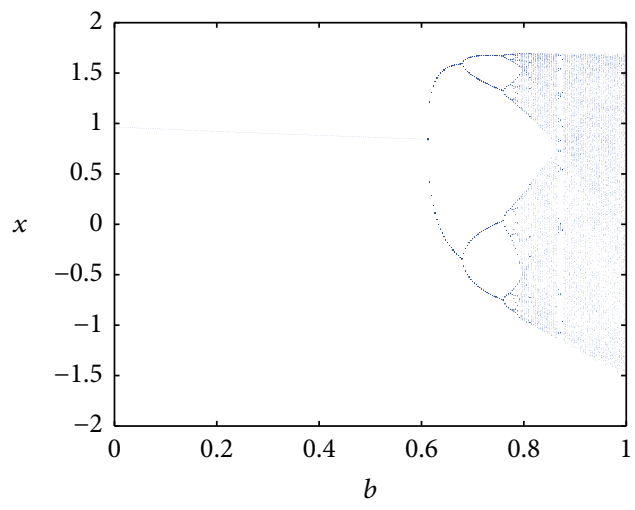

(a)

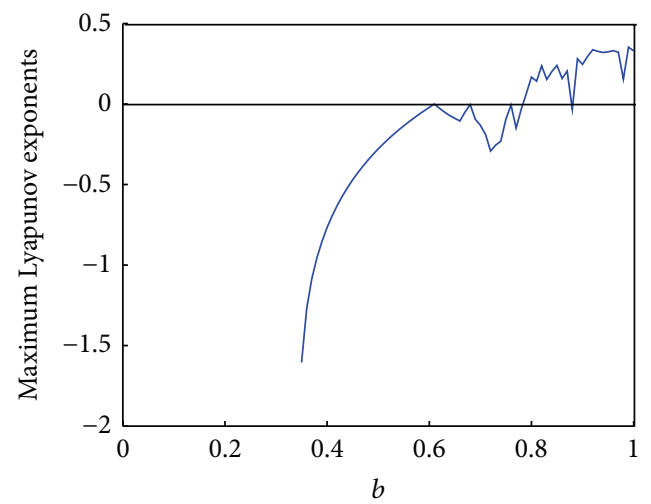

(b)

Figure 6: (a) Bifurcation diagram of map (2) in $(b, x)$ plane for $a=0.5, c=-0.1, d=-0.04$, and $e=0.7$. (b) Maximum Lyapunov exponents corresponding to (a).

For Case (d). The bifurcation diagram of map (2) in $(d, x)$ plane and the corresponding maximum Lyapunov exponents are shown in Figures 10(a) and 10(b), respectively, and the local amplification of (a) for $0.2 \leq d \leq 0.4$ is shown in Figure $10(\mathrm{c})$. we observe that period-doubling bifurcation to chaos and inverse period-doubling bifurcation to chaos, and the Hopf bifurcation occurs at $d \sim-0.75$. The invariant cycle at $d=-0.9$, the two coexisting chaotic attractors at $d=0.28$ and the chaotic attractor at $d=0.4$ are shown in Figures 11(a) and $11(\mathrm{c})$, respectively.

For Case (e). The bifurcation diagram of map (2) in $(e, x)$ plane and the maximum Lyapunov exponents corresponding to (a) are shown in Figures 12(a) and 12(b), respectively. Figures $12(\mathrm{c})-12(\mathrm{~h})$ are the local amplifications of (a). In Figure 12, we observe that the jumping behavior of period3 orbits to period- 1 orbit at $e \sim 0.25$, Hopf bifurcation occurs at $e \sim 0.788$, and the invariant cycle at $e=0.8234$ is shown in Figure 13(a), and the invariant cycle suddenly converges to period- 4 orbits at $e \sim 0.8235$. The region $e \in(0.886,0.9558)$ is the invariant cycle region with complex periodic windows, such as period-21, 17 orbits. The invariant cycle at $a=0.934$ and the forty-one coexisting invariant cycles at $a=0.954$ are shown in Figures 13(b) and 13(c), respectively. For $e \sim 0.9558$, the periodic orbits suddenly become chaotic behaviors. Moreover, the chaotic behaviors suddenly change into a special chaotic behaviors which has 13 small size chaotic attractors as $e$ increases. The chaotic attractor at $e=0.957$ and the thirteen coexisting chaotic attractors at $e=0.959$ are shown in Figures 13(d) and 13(e), respectively. For $e$ 0.9611 , the chaotic behaviors suddenly converge period-13 orbits, which quickly undergo Hopf bifurcation and change into period-13 orbits again. For the region $e \in(0.9633,1.05)$, the period-doubling bifurcation to chaos occurs several times and the chaotic behaviors with complex periodic windows, and the nine coexisting chaotic attractors at $e=0.9725$, the chaotic attractor at $e=0.9901$, the ten coexisting chaotic attractors at $e=1.016$, and the chaotic attractor at $e=1.05$ are shown in Figures 13(f)-13(i), respectively.

\section{Conclusion}

In this paper, the generalized Hénon map with nonconstant Jacobian determinant is investigated. By using the analytic and numerical methods, the existence conditions of fold bifurcation, flip bifurcation, Hopf bifurcation, and Marotto's chaos are obtained. Moreover, we discover some interesting 


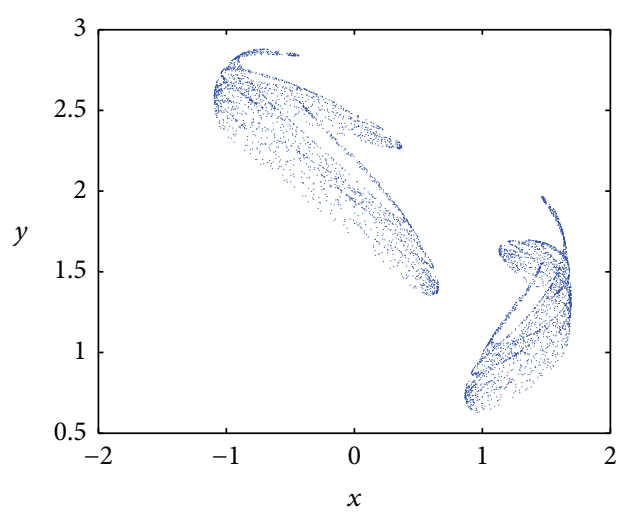

(a)

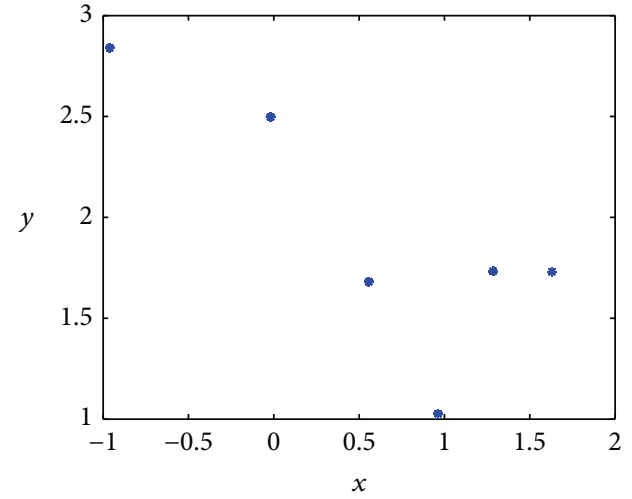

(b)

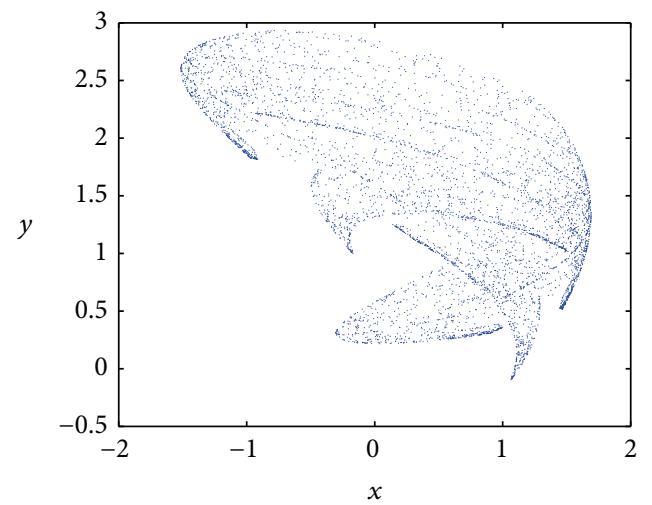

(c)

Figure 7: Phase portraits corresponding to Figure 6: (a) $b=0.85$, (b) $b=0.88$, and (c) $b=1$.

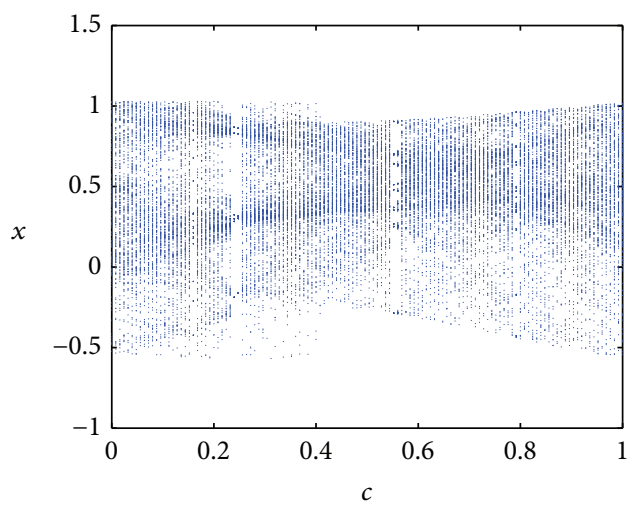

(a)

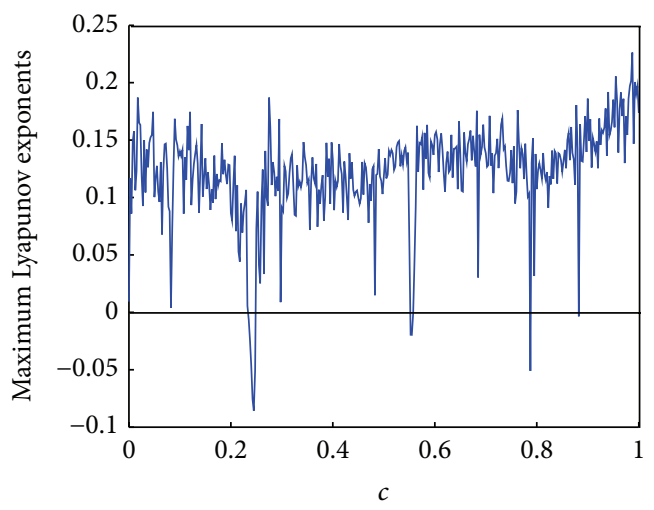

(b)

FIGURE 8: (a) Bifurcation diagram of map (2) in $(c, x)$ plane for $a=0.2, b=1, d=-1.5$, and $e=0.23$. (b) Maximum Lyapunov exponents corresponding to (a).

dynamical behaviors, such as the invariant cycles which are irregular closed graphics, and the six and forty-one coexisting invariant cycles, and the two, six, seven, nine, ten, and thirteen coexisting chaotic attractors, and some kinds of strange chaotic attractors. The numerical simulations show the consistence with the theoretical analysis and exhibit some specific properties which can not be obtained by theoretical analysis.

\section{Appendices}

\section{A. For the Proof of Theorem 3}

The characteristic equation associated with the linearization of map (2) about the fixed point $Z\left(x_{0}, y_{0}\right)$ is given by

$$
\lambda^{2}+p\left(b_{0}\right) \lambda+q\left(b_{0}\right)=0
$$




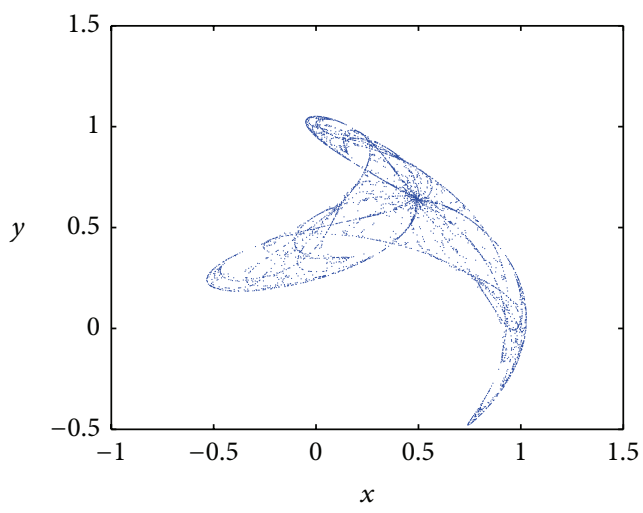

(a)

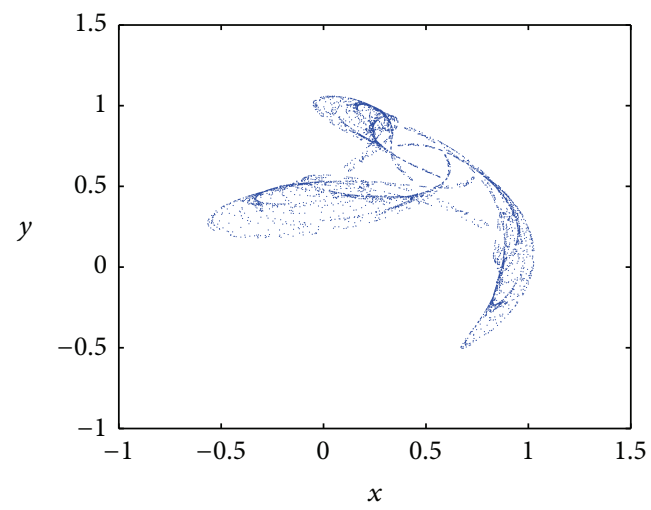

(b)

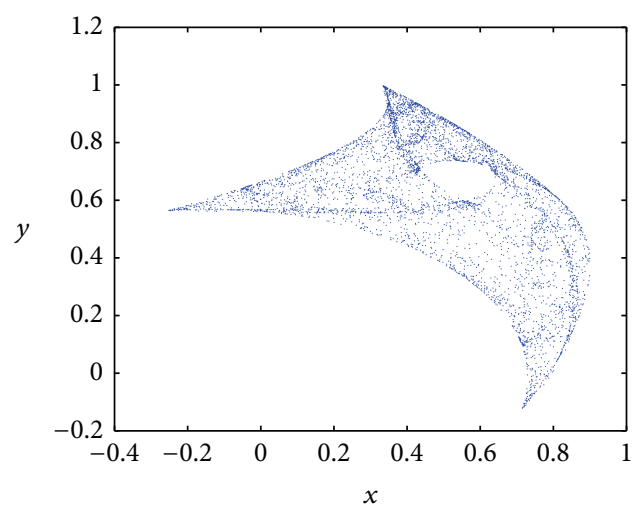

(c)

FIGURE 9: Phase portraits corresponding to Figure 8: (a) $c=0.01$, (b) $c=0.2$, and (c) $c=0.45$.

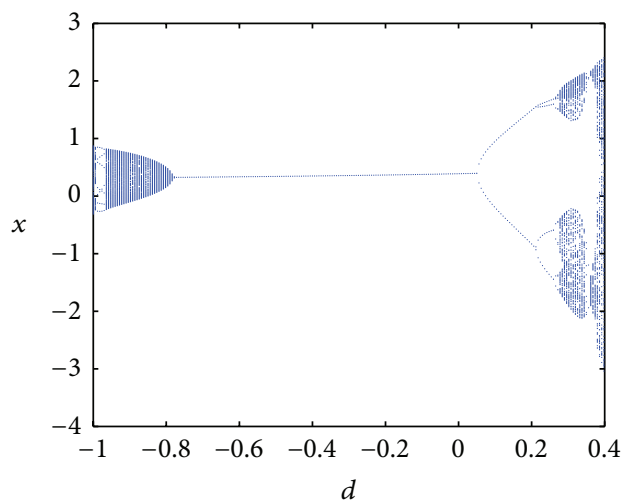

(a)

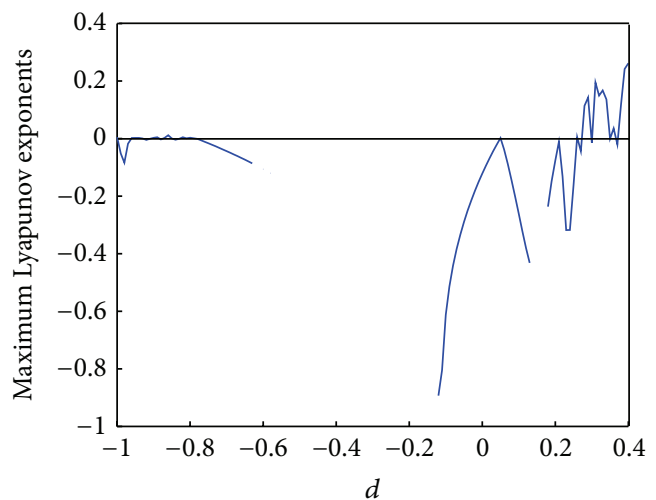

(b)

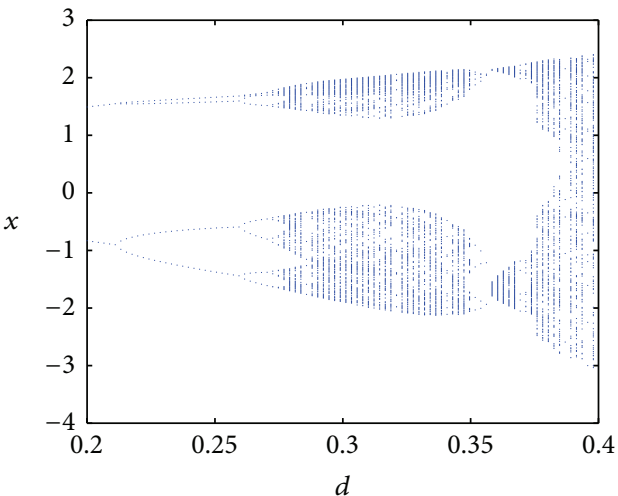

(c)

FIGURE 10: (a) Bifurcation diagram of map (2) in $(d, x)$ plane for $a=-0.3, b=0.9, c=-0.8$, and $e=0.5$. (b) Maximum Lyapunov exponents corresponding to (a). (c) Local amplification of (a). 


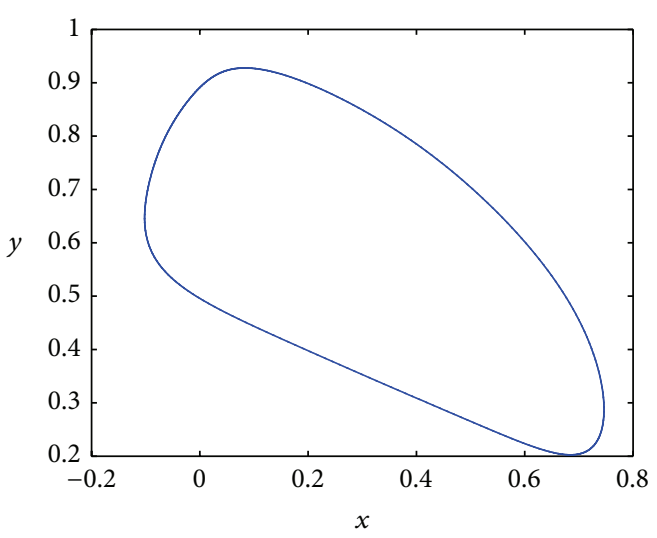

(a)

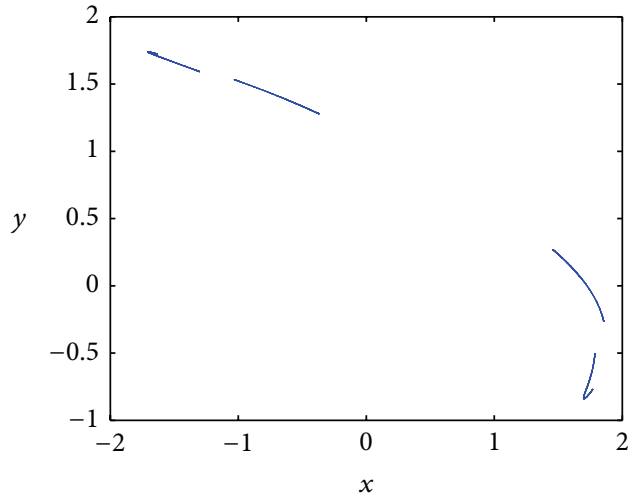

(b)

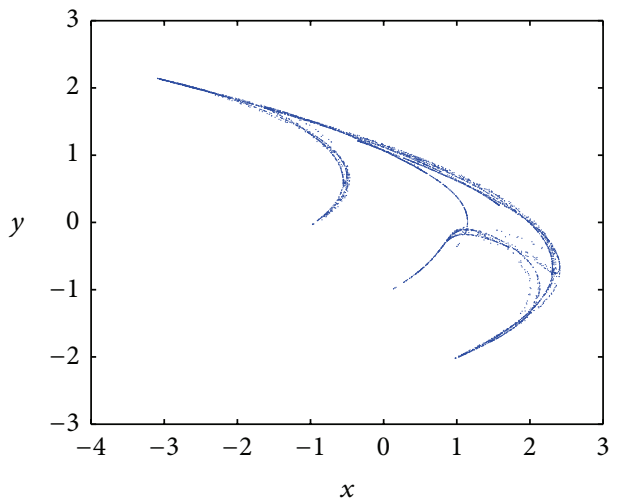

(c)

FIgURE 11: Phase portraits corresponding to Figure 10: (a) $d=-0.9$, (b) $d=0.28$, and (c) $d=0.4$.

where $p\left(b_{0}\right)=2 b x_{0}-c y_{0}-e, q\left(b_{0}\right)=-(2 b e+c) x_{0}-3 d y_{0}^{2}+$ cey $y_{0}-a$.

Supposing that $b_{0}=\left(c x_{0}+3 d y_{0}^{2}+c(1-e) y_{0}+a+e-1\right) / 2(1-$ e) $x_{0}$, the eigenvalues of the fixed point $Z\left(x\left(b_{0}\right), y\left(b_{0}\right)\right)=$ $\left(x_{0}, y_{0}\right)$ are $\lambda_{1}=1$ and $\lambda_{2}=-1-p\left(b_{0}\right)=-1-2 b x_{0}+c y_{0}+e$. For codimension-one bifurcation, it requires $\left|\lambda_{2}\right| \neq 1$, which leads to $b_{0} \neq\left(c y_{0}+e\right) / 2 x_{0}$ and $b_{0} \neq\left(c y_{0}+e-2\right) / 2 x_{0}$.

Let $u=x-x_{0}$, let $v=y-y_{0}$, and let $\widetilde{b}=b-b_{0}$, we transform the fixed point $Z\left(x_{0}, y_{0}\right)$ of map (2) to the origin and consider the parameter $\widetilde{b}$ as a new dependent variable, we can write the map (2) in the following form:

$$
\begin{aligned}
\left(\begin{array}{c}
u \\
\tilde{b} \\
v
\end{array}\right) & \longrightarrow\left(\begin{array}{ccc}
-2 b_{0} x_{0}+c y_{0} & -x_{0}^{2} & a+c x_{0}+3 d y_{0}^{2} \\
0 & 1 & 0 \\
1 & 0 & e
\end{array}\right)\left(\begin{array}{l}
u \\
\tilde{b} \\
v
\end{array}\right) \\
& +\left(\begin{array}{c}
f(u, \widetilde{b}, v) \\
0 \\
0
\end{array}\right),
\end{aligned}
$$

where $f(u, \widetilde{b}, v)=-\widetilde{b} u^{2}-2 x_{0} \widetilde{b} u-b_{0} u^{2}+c u v+d v^{3}+3 d y_{0} v^{2}$.
We construct an invertible matrix

$$
T=\left(\begin{array}{ccc}
1-e & -x_{0}^{2} & a+c x_{0}+3 d y_{0}^{2} \\
0 & 2+2 b_{0} x_{0}-c y_{0}-e & 0 \\
1 & 0 & 1+2 b_{0} x_{0}-c y_{0}
\end{array}\right)
$$

and use the translation $(u, \widetilde{b}, v)^{T}=T(\widetilde{x}, \mu, \tilde{y})^{T}$; then, the map (A.2) becomes

$$
\left(\begin{array}{l}
\tilde{x} \\
\mu \\
\tilde{y}
\end{array}\right) \rightarrow\left(\begin{array}{ccc}
1 & 1 & 0 \\
0 & 1 & 0 \\
0 & 0 & -1-p\left(b_{0}\right)
\end{array}\right)\left(\begin{array}{l}
\tilde{x} \\
\mu \\
\tilde{y}
\end{array}\right)+\left(\begin{array}{c}
f(\tilde{x}, \mu, \tilde{y}) \\
0 \\
g(\tilde{x}, \mu, \tilde{y})
\end{array}\right),
$$

where $f(\tilde{x}, \mu, \tilde{y})=(1 / A)\left\{\left(A \mu / x_{0}^{2}-b_{0}\right)[(1-e) \tilde{x}+\mu-B \tilde{y}]^{2}+\right.$ $\left.\left[2 A \mu / x_{0}+c(\tilde{x}+\tilde{y})\right][(1-e) \tilde{x}+\mu-B \tilde{y}]+d(\tilde{x}+\tilde{y})^{3}+3 d y_{0}(\tilde{x}+\tilde{y})^{2}\right\}$, $g(\tilde{x}, \mu, \tilde{y})=-f(\tilde{x}, \mu, \tilde{y}), A=2 b_{0} x_{0}-c y_{0}-e+2$ and $B=$ $2 b_{0} x_{0}-c y_{0}+1$.

By center manifold theory, the stability of $(\tilde{x}, \tilde{y})=(0,0)$ near $\mu=0$ can be determined by studying a one-parameter 


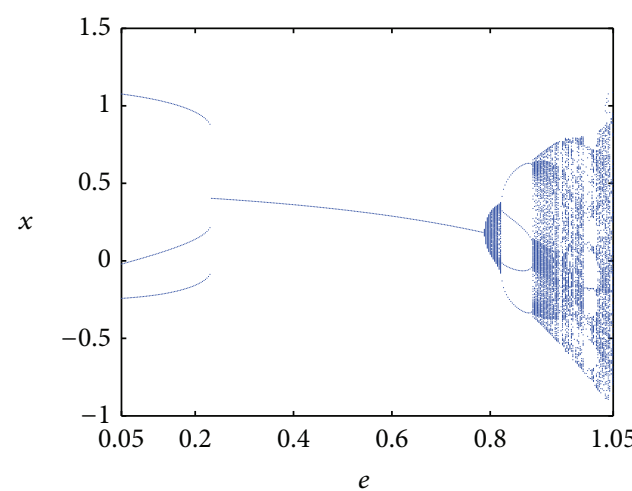

(a)

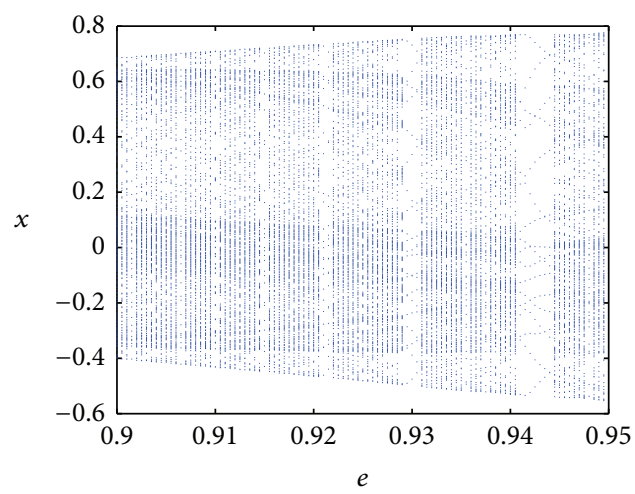

(c)

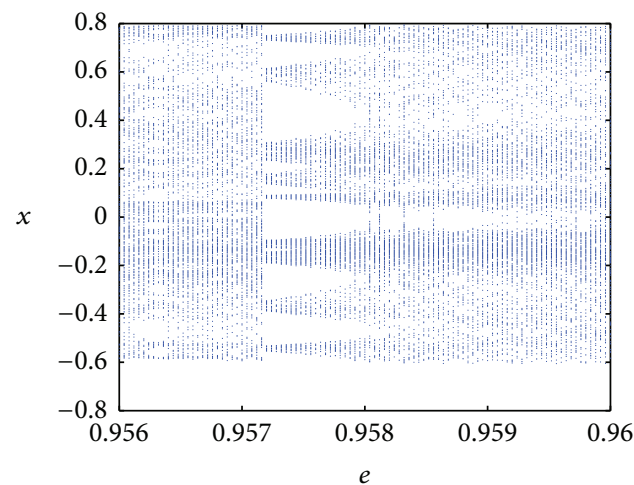

(e)

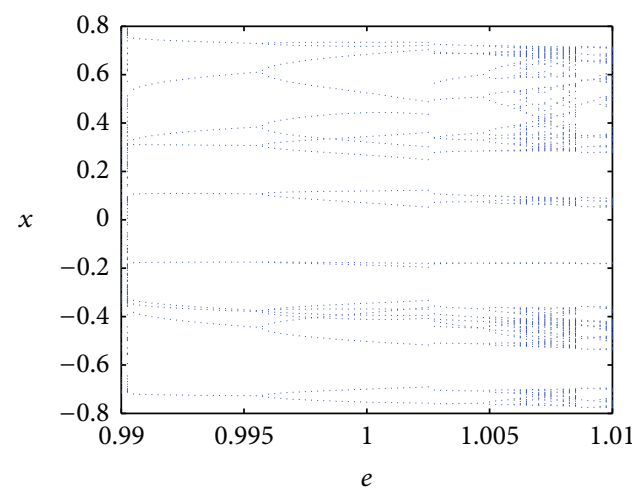

(g)

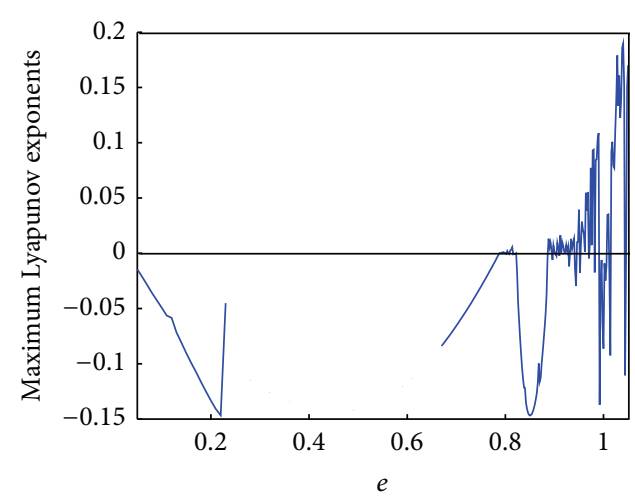

(b)

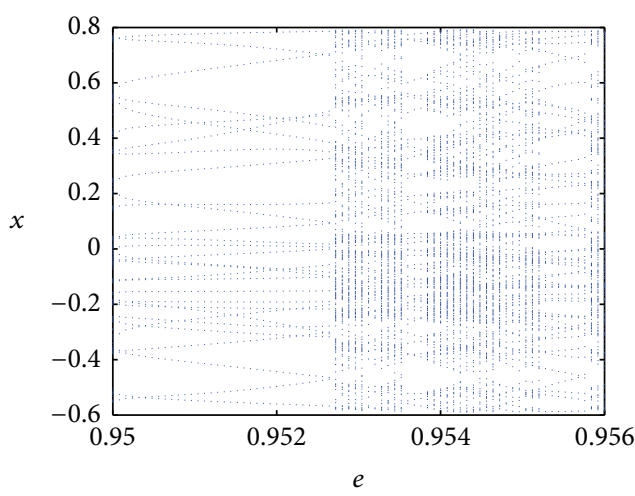

(d)

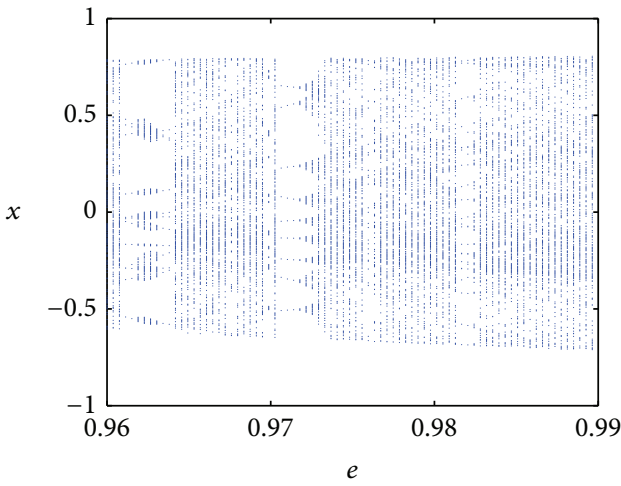

(f)

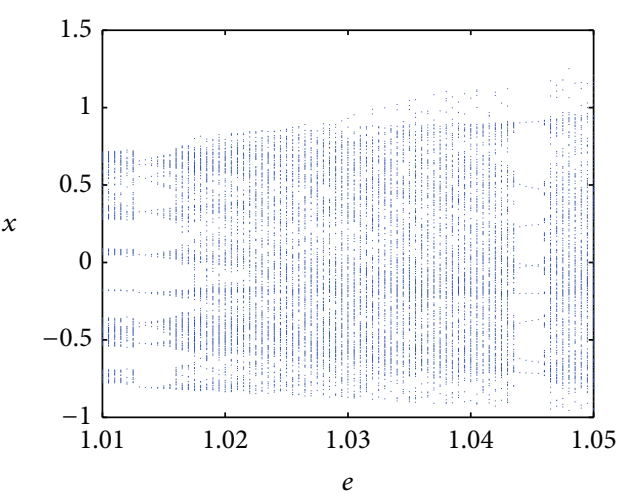

(h)

FIGURE 12: (a) Bifurcation diagram of map (2) in $(e, x)$ plane for $a=-0.4, b=1.1, c=-0.6$, and $d=-0.55$. (b) Maximum Lyapunov exponents corresponding to (a). (c)-(h) Local amplification of (a) for (c) $0.9 \leq e \leq 0.95$, (d) $0.95 \leq e \leq 0.956$, (e) $0.956 \leq e \leq 0.96$, (f) $0.96 \leq e \leq 0.99$, (g) $0.99 \leq e \leq 1.01$, and (h) $1.01 \leq e \leq 1.05$. 


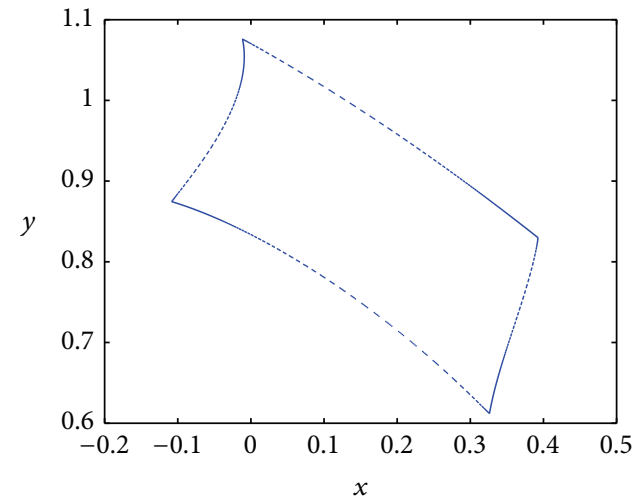

(a)

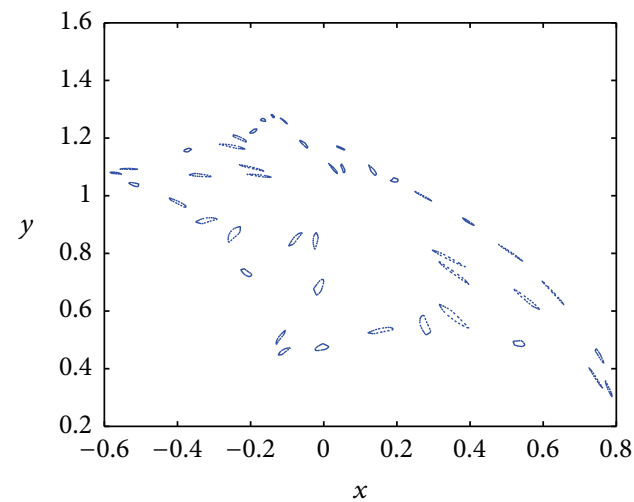

(c)

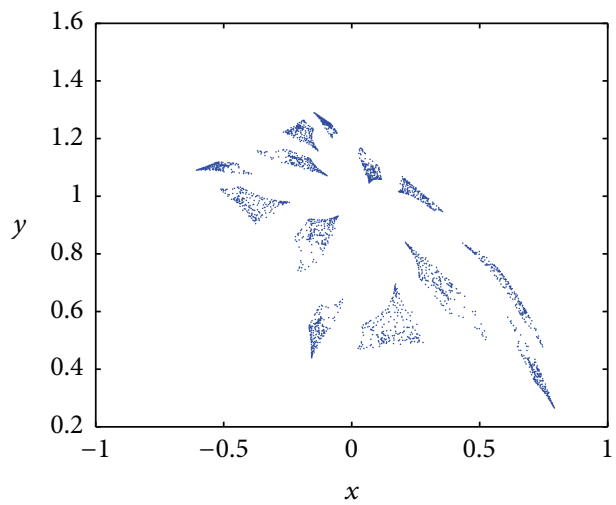

(e)

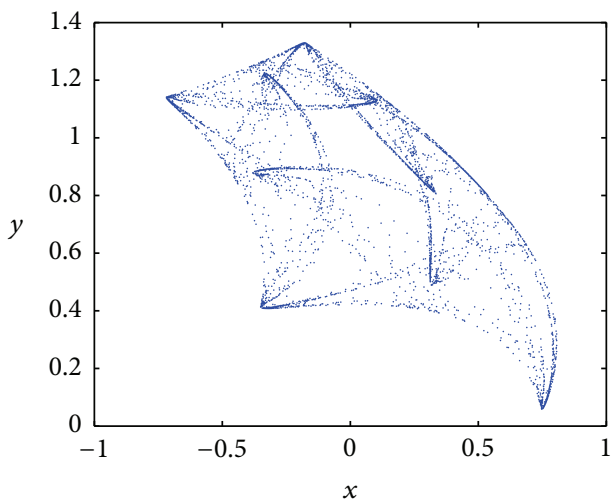

(g)

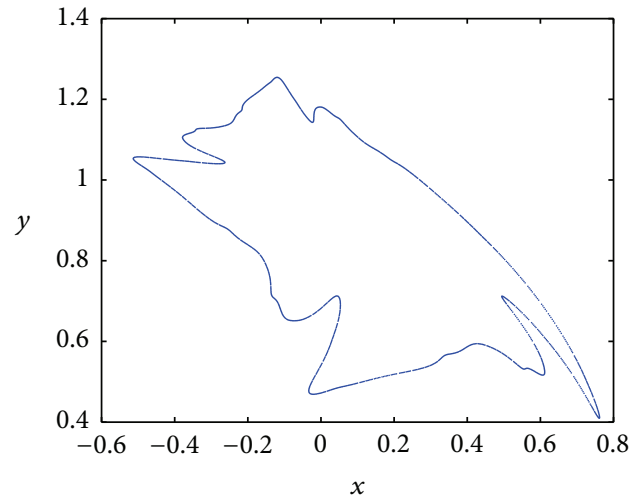

(b)

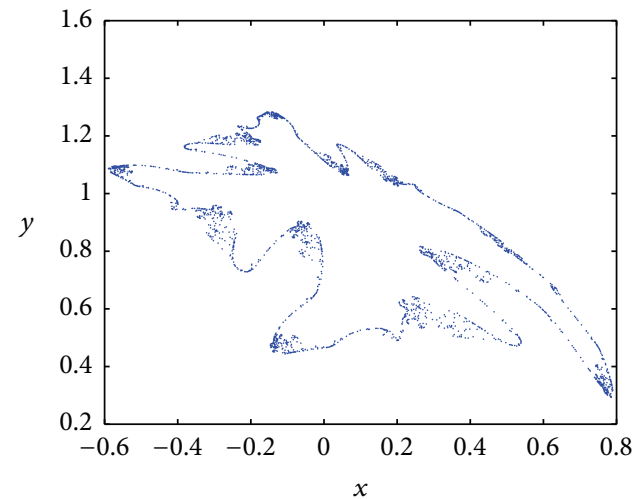

(d)

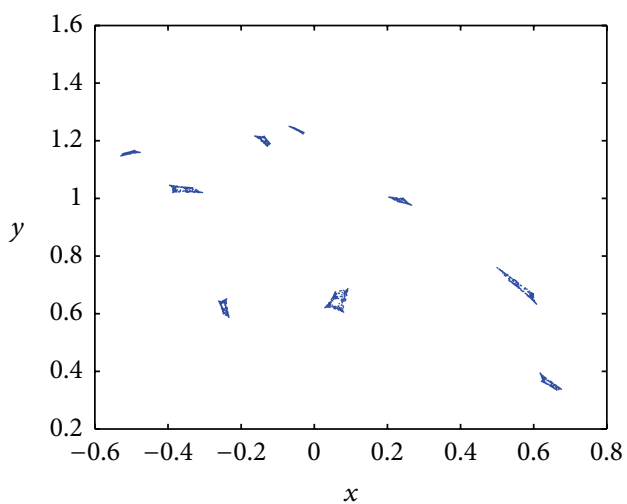

(f)

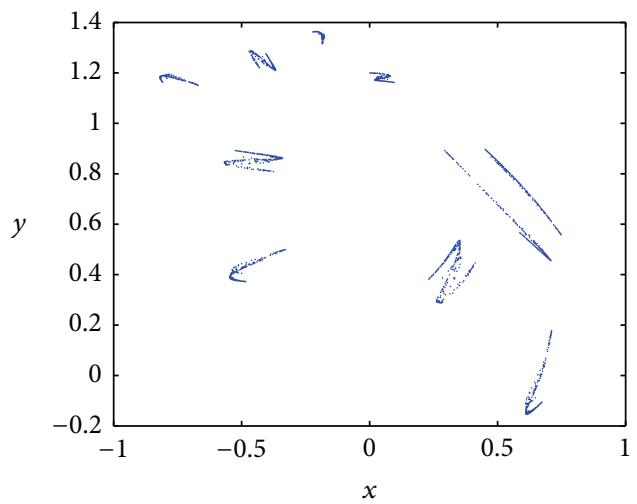

(h)

FIgUre 13: Continued. 


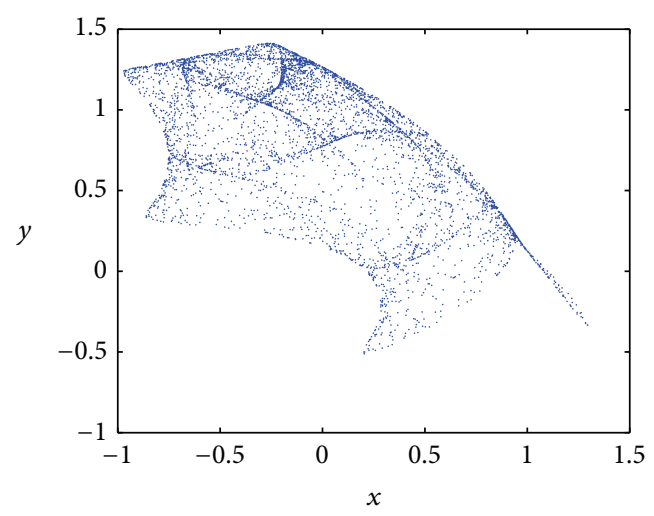

(i)

FiguRE 13: Phase portraits corresponding to Figure 12: (a) $e=0.8234$, (b) $e=0.934$, (c) $e=0.954$, (d) $e=0.957$, (e) $e=0.959$, (f) $e=0.9725$, (g) $e=0.9901$, (h) $e=1.016$, and (i) $e=1.05$.

family of equation on a center manifold, which can be represented as follows:

$W^{c}(0)$

$=\left\{(\tilde{x}, \mu, \tilde{y}) \in \mathbb{R}^{3} \mid \tilde{y}=h(\tilde{x}, \mu), h(0,0)=0, D h(0,0)=0\right\}$,

for $\tilde{x}$ and $\mu$ sufficiently small.

Assume that a center manifold is of the form $h(\tilde{x}, \mu)=$ $\alpha \widetilde{x}^{2}+\beta \widetilde{x} \mu+\gamma \mu^{2}+O\left((|\widetilde{x}|+|\mu|)^{3}\right)$, the center manifold must satisfy

$$
\begin{aligned}
N(h(\tilde{x}, \mu))= & h(\tilde{x}+\mu+f(\tilde{x}, \mu, h(\tilde{x}, \mu)), \mu) \\
& -\left(-1-p\left(b_{0}\right)\right) h(\tilde{x}, \mu)-g(\tilde{x}, \mu, h(\tilde{x}, \mu)) \\
= & 0 .
\end{aligned}
$$

Since only the terms with orders lower than 3 for map (A.4) are concerned, the map restricted to the center manifold directly from (A.4) can be given by

$$
\begin{aligned}
\tilde{x} & \longrightarrow \tilde{f}(\tilde{x}, \mu) \\
& =\tilde{x}+\mu+\alpha \tilde{x}^{2}+\beta \tilde{x} \mu+\gamma \mu^{2}+O\left((|\tilde{x}|+|\mu|)^{3}\right),
\end{aligned}
$$

where

$$
\begin{gathered}
\alpha=\frac{1}{A^{2}}\left[b_{0}(1-e)^{2}-c(1-e)-3 d y_{0}\right], \\
\beta=\frac{1}{A^{3}}\left[-2 b_{0}(1-e)^{2}+\left(2 b_{0} A-\frac{2 A^{2}}{x_{0}}+2 c\right)(1-e)\right. \\
\left.+6 d y_{0}-c A\right],
\end{gathered}
$$

$$
\begin{array}{r}
\gamma=\frac{1}{A^{4}}\left[b_{0}(2-A)(1-e)^{2}+\left(c A-2 b_{0} A+\frac{2 A^{2}}{x_{0}}-2 c\right)\right. \\
\left.\cdot(1-e)-6 d y_{0}+3 d A y_{0}+c A+b_{0} A^{2}-\frac{2 A^{3}}{x_{0}}\right] .
\end{array}
$$

Since $\tilde{f}(0,0)=0,(\partial \tilde{f} / \partial \tilde{x})(0,0)=1,(\partial \tilde{f} / \partial \mu)(0,0)=1$, and $\left(\partial^{2} \tilde{f} / \partial \widetilde{x}^{2}\right)(0,0)=2 \alpha \neq 0$, the fixed point $(\widetilde{x}, \mu)=(0,0)$ is a fold bifurcation point for map (A.4) and the number of fixed points is changed at $(\widetilde{x}(\mu), \tilde{y}(\mu))=(0,0)$ as $\mu=0$.

\section{B. For the Proof of Theorem 4}

Supposing that $b^{*}=\left(-c x_{0}-3 d y_{0}^{2}+c(1+e) y_{0}-a+e+1\right) / 2(1+$ e) $x_{0}$, the eigenvalues of the fixed point $Z\left(x\left(b^{*}\right), y\left(b^{*}\right)\right)=$ $\left(x_{0}, y_{0}\right)$ are $\lambda_{1}=-1$ and $\lambda_{2}=1-p\left(b^{*}\right)=-2 b^{*} x_{0}+c y_{0}+e+1$.

The condition $\left|\lambda_{2}\right| \neq 1$ leads to $b^{*} \neq\left(c y_{0}+e+1\right) / 2 x_{0}$ and $b^{*} \neq\left(c y_{0}+e+3\right) / 2 x_{0}$, and the condition $q\left(b^{*}\right) \neq 0$ leads to $b^{*} \neq\left(-c x_{0}-3 d y_{0}^{2}+c e y_{0}-a\right) / 2 e x_{0}$.

Let $u=x-x_{0}$, let $v=y-y_{0}$, and let $\widetilde{b}^{*}=b-b^{*}$; we transform the fixed point $Z\left(x_{0}, y_{0}\right)$ of map (2) to the origin and consider $\widetilde{b}^{*}$ as a new dependent variable; then, map (2) becomes

$$
\begin{aligned}
\left(\begin{array}{c}
u \\
\tilde{b}^{*} \\
v
\end{array}\right) \longrightarrow & \left(\begin{array}{ccc}
-2 b^{*} x_{0}+c y_{0} & -x_{0}^{2} & a+c x_{0}+3 d y_{0}^{2} \\
0 & 1 & 0 \\
1 & 0 & e
\end{array}\right) \\
& \cdot\left(\begin{array}{c}
u \\
\tilde{b}^{*} \\
v
\end{array}\right)+\left(\begin{array}{c}
f\left(u, \widetilde{b}^{*}, v\right) \\
0 \\
0
\end{array}\right),
\end{aligned}
$$

where $f\left(u, \widetilde{b}^{*}, v\right)=-\widetilde{b}^{*} u^{2}-2 x_{0} \widetilde{b}^{*} u-b^{*} u^{2}+c u v+d v^{3}+3 d y_{0} v^{2}$. 
We construct an invertible matrix

$$
T=\left(\begin{array}{ccc}
e+1 & -1 & 2 b^{*} x_{0}-c y_{0}-1 \\
0 & \frac{2 b^{*} x_{0}-c y_{0}-e-2}{x_{0}^{2}} & 0 \\
-1 & 0 & -1
\end{array}\right)
$$

and use the translation $\left(u, \widetilde{b}^{*}, v\right)^{T}=T(\tilde{x}, \mu, \tilde{y})^{T}$; map (B.1) becomes

$$
\left(\begin{array}{c}
\tilde{x} \\
\mu \\
\tilde{y}
\end{array}\right) \longrightarrow\left(\begin{array}{ccc}
-1 & 1 & 0 \\
0 & -1 & 0 \\
0 & 0 & 1-p\left(b^{*}\right)
\end{array}\right)\left(\begin{array}{l}
\tilde{x} \\
\mu \\
\tilde{y}
\end{array}\right)+\left(\begin{array}{c}
f(\tilde{x}, \mu, \tilde{y}) \\
0 \\
g(\tilde{x}, \mu, \tilde{y})
\end{array}\right)
$$

where $f(\tilde{x}, \mu, \tilde{y})=\left(1 / A_{1}\right)\left\{\left(A_{1} / x_{0}^{2}\right) \mu\left[(e+1) \tilde{x}-\mu+B_{1} \tilde{y}\right]^{2}+\right.$ $2\left(A_{1} / x_{0}\right) \mu\left[(e+1) \tilde{x}-\mu+B_{1} \tilde{y}\right]+b^{*}\left[(e+1) \tilde{x}-\mu+B_{1} \tilde{y}\right]^{2}+c[(e+$ 1) $\left.\left.\tilde{x}-\mu+B_{1} \tilde{y}\right](\tilde{x}+\tilde{y})+d(\tilde{x}+\tilde{y})^{3}-3 d y_{0}(\tilde{x}+\tilde{y})^{2}\right\}, g(\tilde{x}, \mu, \tilde{y})=$ $-f(\tilde{x}, \mu, \tilde{y}), A_{1}=2 b^{*} x_{0}-c y_{0}-e-2$, and $B_{1}=2 b^{*} x_{0}-c y_{0}-1$.

We again apply the center manifold theorem to determine the nature of the bifurcation of the fixed point $(\widetilde{x}, \widetilde{y})=(0,0)$ at $\mu=0$. There exists a center manifold for map (B.3), which can be represented as follows:

$$
\begin{aligned}
& W^{c}(0) \\
& =\left[(\tilde{x}, \mu, \tilde{y}) \in \mathbb{R}^{3} \mid \tilde{y}=h^{*}(\tilde{x}, \mu),\right. \\
& \left.h^{*}(0,0)=0, D h^{*}(0,0)=0\right],
\end{aligned}
$$

for $\tilde{x}$ and $\mu$ sufficiently small.

Assuming a center manifold of the form $h^{*}(\widetilde{x}, \mu)=\alpha^{*} \tilde{x}^{2}+$ $\beta^{*} \tilde{x} \mu+\gamma^{*} \mu^{2}+O\left((|\tilde{x}|+|\mu|)^{3}\right)$, the center manifold must satisfy

$$
\begin{aligned}
N(h(\tilde{x}, \mu))= & h(-\tilde{x}+\mu+f(\tilde{x}, \mu, h(\tilde{x}, \mu)), \mu) \\
& -\left(1-p\left(b^{*}\right)\right) h(\tilde{x}, \mu)-g(\widetilde{x}, \mu, h(\widetilde{x}, \mu))=0 .
\end{aligned}
$$

By the approximate calculation for center manifold, the coefficients $\alpha^{*}=-\left(1 / A_{1}\left(A_{1}+2\right)\right)\left[b^{*}(e+1)^{2}+c(e+1)+3 d y_{0}\right]$, $\beta^{*}=\left(1 /\left(A_{1}+1\right)\right)\left[\left(2 b^{*} / A_{1}\right)(e+1)-2 / x_{0}+c / A_{1}+2 \alpha^{*}\right]$, $\gamma^{*}=\left(1 /\left(A_{1}+2\right)\right)\left[-b^{*} / A_{1}+2 / x_{0}-\alpha^{*}\right]$, and the map restricted to the center manifold is given by

$$
\begin{aligned}
\tilde{x} \longrightarrow F(\tilde{x}, \mu)= & -\tilde{x}+\mu+\alpha^{*} \tilde{x}^{2}+\beta^{*} \tilde{x} \mu+\gamma^{*} \mu^{2}+k_{1} \tilde{x}^{3} \\
& +k_{2} \tilde{x}^{2} \mu+k_{3} \tilde{x} \mu^{2}+k_{4} \mu^{3}+O\left((|\widetilde{x}|+|\mu|)^{4}\right),
\end{aligned}
$$

where

$$
\begin{aligned}
k_{1}= & \frac{1}{A_{1}}\left[2 B_{1} b^{*}(e+1)+B_{1} c-6 d y_{0}\right] \alpha^{*}+\frac{d}{A_{1}}, \\
k_{2}= & \frac{B_{1}}{A_{1}}\left(-2 b^{*}+\frac{2 A_{1}}{x_{0}}-\frac{c}{B_{1}}\right) \alpha^{*} \\
& +\frac{1}{A_{1}}\left[b^{*}(e+1)^{2}+c(e+1)-9 d y_{0}\right. \\
& \left.+2 B_{1} b^{*}(e+1)+B_{1} c-c\right] \beta^{*}, \\
k_{3}= & \frac{1}{A_{1}}\left[-2 b^{*}(e+1)+\frac{2 A_{1}}{x_{0}}-c-2 B_{1} b^{*}+\frac{2 A_{1} B_{1}}{x_{0}}-c\right] \beta^{*} \\
& +\frac{1}{A_{1}}\left[2 B_{1} b^{*}(e+1)+B_{1} c+c-6 d y_{0}\right] \gamma^{*}, \\
k_{4}= & \left(\frac{b^{*}}{A_{1}}-\frac{2}{x_{0}}\right) \beta^{*}+\left(-\frac{2 B_{1}}{A_{1}} b^{*}+\frac{2 B_{1}}{x_{0}}-\frac{c}{A_{1}}\right) \gamma^{*} .
\end{aligned}
$$

In order for map (B.6) to undergo a flip bifurcation, it is also required that

$$
\begin{aligned}
\alpha_{1}= & \left.\left(\frac{\partial F}{\partial \mu} \frac{\partial^{2} F}{\partial \widetilde{x}^{2}}+2 \frac{\partial^{2} F}{\partial \widetilde{x} \partial \mu}\right)\right|_{(0,0)} \\
= & \frac{2}{A_{1}\left(A_{1}+1\right)}\left[2 b^{*}(e+1)-\frac{2 A_{1}}{x_{0}}+c\right] \\
& +\frac{2\left(A_{1}+3\right)}{A_{1}+1} \alpha^{*} \neq 0, \\
\alpha_{2}= & \left.\left(\frac{1}{2}\left(\frac{\partial^{2} F}{\partial \widetilde{x}^{2}}\right)^{2}+\frac{1}{3}\left(\frac{\partial^{3} F}{\partial \widetilde{x}^{3}}\right)\right)\right|_{(0,0)} \\
= & 2\left(\alpha^{*}\right)^{2}+\frac{2 \alpha^{*}}{A_{1}}\left[2 B_{1} b^{*}(e+1)+B_{1} c-6 d y_{0}\right]+\frac{d}{A_{1}} \neq 0 .
\end{aligned}
$$

\section{For the Proof of Theorem 5}

The eigenvalues of the characteristic equation at fixed point $Z(x(b), y(b))$ are $\lambda_{1,2}=\left(-p(b) \pm \sqrt{p^{2}(b)-4 q(b)}\right) / 2$, where $p^{2}(b)-4 q(b)=4 x_{0}^{2} b^{2}+4 x_{0}\left(e-c y_{0}\right) b+\left(c^{2}+12 d\right) y_{0}^{2}+4 c x_{0}-$ $2 c e y_{0}+4 a+e^{2}$. The eigenvalues $\lambda_{1,2}$ are complex conjugate for $p^{2}(b)-4 q(b)<0$, which leads to

$$
\begin{gathered}
\Delta^{\prime}=-x_{0}^{2}\left(3 d y_{0}^{2}+c x_{0}+a\right)>0, \\
\frac{-x_{0}\left(e-c y_{0}\right)-2 \sqrt{\Delta^{\prime}}}{2 x_{0}^{2}}<b<\frac{-x_{0}\left(e-c y_{0}\right)+2 \sqrt{\Delta^{\prime}}}{2 x_{0}^{2}} .
\end{gathered}
$$

Assuming that

$$
\bar{b}=\frac{-3 d y_{0}^{2}+c\left(x_{0}-e y_{0}\right)+a+1}{2 e x_{0}},
$$

we have $q(\bar{b})=1$. 
We translate the fixed point $Z(x(\bar{b}), y(\bar{b}))=\left(x_{0}, y_{0}\right)$ of map (2) to the origin by using the translation $u=x-x(\bar{b})$, $v=y-y(\bar{b})$; map (2) becomes

$$
\begin{aligned}
& \left(\begin{array}{l}
u \\
v
\end{array}\right) \\
& \longrightarrow\left(\begin{array}{c}
a v-b u^{2}-2 b x_{0} u+c u v+c y_{0} u+c x_{0} v+d v^{3}+3 d y_{0} v^{2}+3 d y_{0}^{2} v \\
u+e v
\end{array}\right) .
\end{aligned}
$$

For $b=\bar{b}$, the eigenvalues of the matrix associated with the linearized map (2) at fixed point $(0,0)$ are complex conjugate with modulus 1 which are written as

$$
\lambda, \bar{\lambda}=-\frac{p(\bar{b})}{2} \pm \frac{i}{2} \sqrt{4 q(\bar{b})-p^{2}(\bar{b})}=m \pm i n,
$$

where $p(\bar{b})=2 \bar{b} x_{0}-c y_{0}-e$ and $q(\bar{b})=-(2 \bar{b} e+c) x_{0}-3 d y_{0}^{2}+$ $c e y_{0}-a$.

Under the conditions (C.1)-(C.3), there are $|\lambda|=\sqrt{q(\bar{b})}$ and $k=\left.(d|\lambda(b)| / d b)\right|_{b=\bar{b}}=-e x_{0} \neq 0$. In addition, if $p(\bar{b}) \neq$ 0,1 , which leads to $\bar{b} \neq\left(c y_{0}+e\right) / 2 a x_{0}$ and $\bar{b} \neq\left(c y_{0}+e+\right.$ 1)/2ax 0 , then we have $\lambda^{n}(\bar{b}) \neq 1, n=1,2,3,4$.

Let $T=\left(\begin{array}{cc}m-e & n \\ 1 & 0\end{array}\right)$ and use the translation $\left(\begin{array}{l}u \\ v\end{array}\right)=T\left(\begin{array}{l}x \\ y\end{array}\right)$; map (C.4) becomes

$$
\left(\begin{array}{l}
x \\
y
\end{array}\right) \longrightarrow\left(\begin{array}{cc}
m & n \\
-n & m
\end{array}\right)\left(\begin{array}{l}
x \\
y
\end{array}\right)+\left(\begin{array}{c}
0 \\
g(x, y)
\end{array}\right)
$$

where $g(x, y)=-(b / n)[(m-e) x+n y]^{2}+(c / n)[(m-e) x+$ $n y] x+(d / n) x^{3}+\left(3 d y_{0} / n\right) x^{2}$. Let $r=(1 / n)\left[-b(m-e)^{2}+\right.$ $\left.c(m-e)+3 d y_{0}\right]$ and let $s=-2 b(m-e)+c$; then, $g(x, y)=$ $(d / n) x^{3}+r x^{2}+s x y-b n y^{2}$.

Notice that map (C.6) is exactly in the form on the center manifold, in which the coefficient $l$ (see [18]) is given by

$$
l=-\operatorname{Re}\left[\frac{(1-2 \lambda) \bar{\lambda}^{2}}{1-\lambda} \xi_{11} \xi_{20}\right]-\frac{1}{2}\left|\xi_{11}\right|^{2}-\left|\xi_{02}\right|^{2}+\operatorname{Re}\left(\bar{\lambda} \xi_{21}\right),
$$

where $\xi_{20}=(1 / 8)\left[f_{x x}-f_{y y}+2 g_{x y}+i\left(g_{x x}-g_{y y}-2 f_{x y}\right)\right]$, $\xi_{11}=(1 / 4)\left[\left(f_{x x}+f_{y y}\right)+i\left(g_{x x}+g_{y y}\right)\right], \xi_{02}=(1 / 8)\left[\left(f_{x x}-\right.\right.$ $\left.\left.f_{y y}-2 g_{x y}\right)+i\left(g_{x x}-g_{y y}+2 f_{x y}\right)\right]$, and $\xi_{21}=(1 / 16)\left[\left(f_{x x x}+\right.\right.$ $\left.\left.f_{x y y}+g_{x x y}+g_{y y y}\right)+i\left(g_{x x x}+g_{x y y}-f_{x x y}-f_{y y y}\right)\right]$.

Thus, some complicated calculations give

$$
\begin{aligned}
l= & -\frac{3}{32}\left(\frac{6 d}{n} x+2 r\right)^{2}+\frac{n}{16}\left[\frac{s}{2(1-m)}+b\right]\left(\frac{6 d}{n} x+2 r\right) \\
& -\frac{b s n^{2}}{16(1-m)}-\frac{s^{2}}{16}-\frac{3}{8} d \neq 0 .
\end{aligned}
$$

\section{Conflict of Interests}

The author declares that there is no conflict of interests regarding the publication of this paper.

\section{Acknowledgments}

The author would like to thank the reviewers for their helpful comments and suggestions. This work was supported by Hunan Provincial Natural Science Foundation of China (no. 13JJ4088 and no. 14JJ3114), the General Project of Hunan Provincial Education Department (no. 14C1191), and the National Natural Science Foundation of China (no. 11301551, no. 11426221, and no. 11426223).

\section{References}

[1] M. Hénon, "A two-dimensional mapping with a strange attractor," Communications in Mathematical Physics, vol. 50, no. 1, pp. 69-77, 1976.

[2] S. D. Feit, "Characteristic exponents and strange attractors," Communications in Mathematical Physics, vol. 61, no. 3, pp. 249260, 1978.

[3] F. R. Marotto, "Chaotic behavior in the Hénon mapping," Communications in Mathematical Physics, vol. 68, no. 2, pp. 187194, 1979.

[4] J. H. Curry, "On the Hénon transformation," Communications in Mathematical Physics, vol. 68, no. 2, pp. 129-140, 1979.

[5] S. Smale, "Differentiable dynamical systems," Bulletin of the American Mathematical Society, vol. 73, pp. 747-817, 1967.

[6] L. Mora and M. Viana, "Abundance of strange attractors," Acta Mathematica, vol. 171, no. 1, pp. 1-71, 1993.

[7] M. Sonis, "Once more on Hénon map: analysis of bifurcations," Chaos, Solitons and Fractals, vol. 7, no. 12, pp. 2215-2234, 1996.

[8] Y. Cao, “The nonwandering set of some Hénon map," Chinese Science Bulletin, vol. 44, no. 7, pp. 590-594, 1999.

[9] A. C. J. Luo and Y. Guo, "Complete bifurcation behaviors of a henon map," Dynamical Systems: Discontinuity, Stochasticity and Time-Delay, pp. 37-47, 2010.

[10] Y. Zhang, "Switching-induced Wada basin boundaries in the Hénon map," Nonlinear Dynamics, vol. 73, no. 4, pp. 2221-2229, 2013.

[11] R. Brown, "Horseshoes in the measure-preserving Hénon map," Ergodic Theory and Dynamical Systems, vol. 15, no. 6, pp. 10451059, 1995.

[12] U. Kirchgraber and D. Stoffer, "Transversal homoclinic points of the Hénon map," Annali di Matematica Pura ed Applicata, vol. 185, no. 5, pp. S187-S204, 2006.

[13] E. Jensen, "A new construction of the unstable manifold for the measure-preserving Hénon map," Proceedings of the American Mathematical Society, vol. 136, no. 1, pp. 181-192, 2008.

[14] S. V. Gonchenko and V. S. Gonchenko, On Andronov-Hopf Bifurcations of Two-Dimensional Diffeomorphisms with Homoclinic Tangencies, WIAS, Berlin, Germany, 2000.

[15] S. V. Gonchenko, L. P. Shilnikov, and O. V. Stenkin, "On newhouse regions with infinitely many stable and unstable tori," in Proceedings of the International Conference on Progress in Nonlinear Science, vol. 1, pp. 80-102, RAS Institute of Applied Physics, Nizhny Nogorod, Russia, 2002.

[16] S. V. Gonchenko, D. V. Turaev, and L. P. Shil'nikov, "On dynamic properties of diffeomorphisms with homoclinic tangency," Journal of Mathematical Sciences, vol. 126, no. 4, pp. 1317-1343, 2005.

[17] D. V. Turaev, "On dimension of non-local bifurcational problems," International Journal of Bifurcation and Chaos in Applied Sciences and Engineering, vol. 6, no. 5, pp. 919-948, 1996. 
[18] J. Guckenheimer and P. Holmes, Nonlinear Oscillations, Dynamical Systems, and Bifurcations of Vector Fields, Springer, New York, NY, USA, 1983.

[19] F. R. Marotto, "Snap-back repellers imply chaos in $\mathbb{R}^{n}$," Journal of Mathematical Analysis and Applications, vol. 63, no. 1, pp. 199223, 1978. 


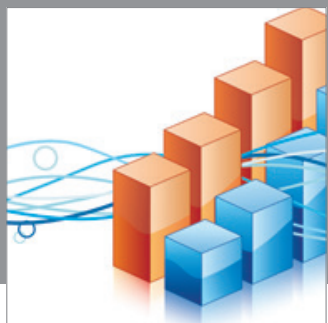

Advances in

Operations Research

mansans

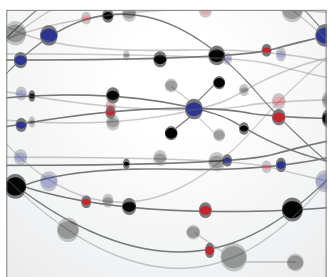

The Scientific World Journal
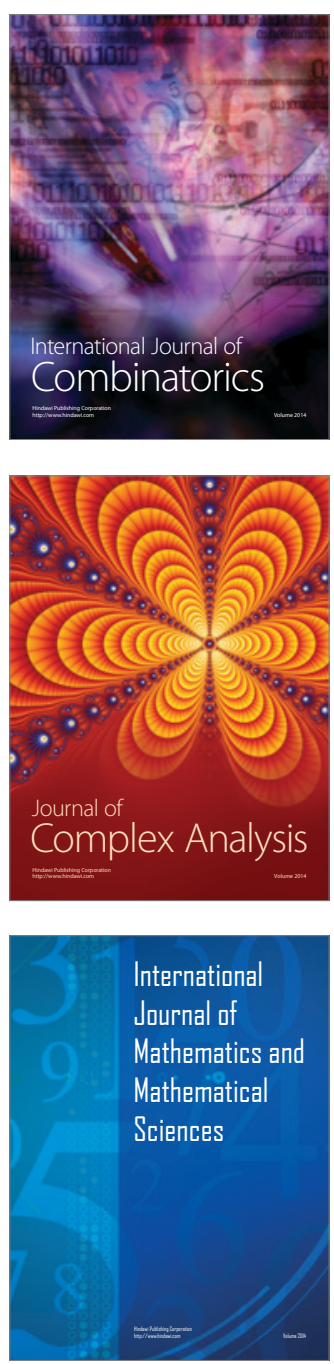
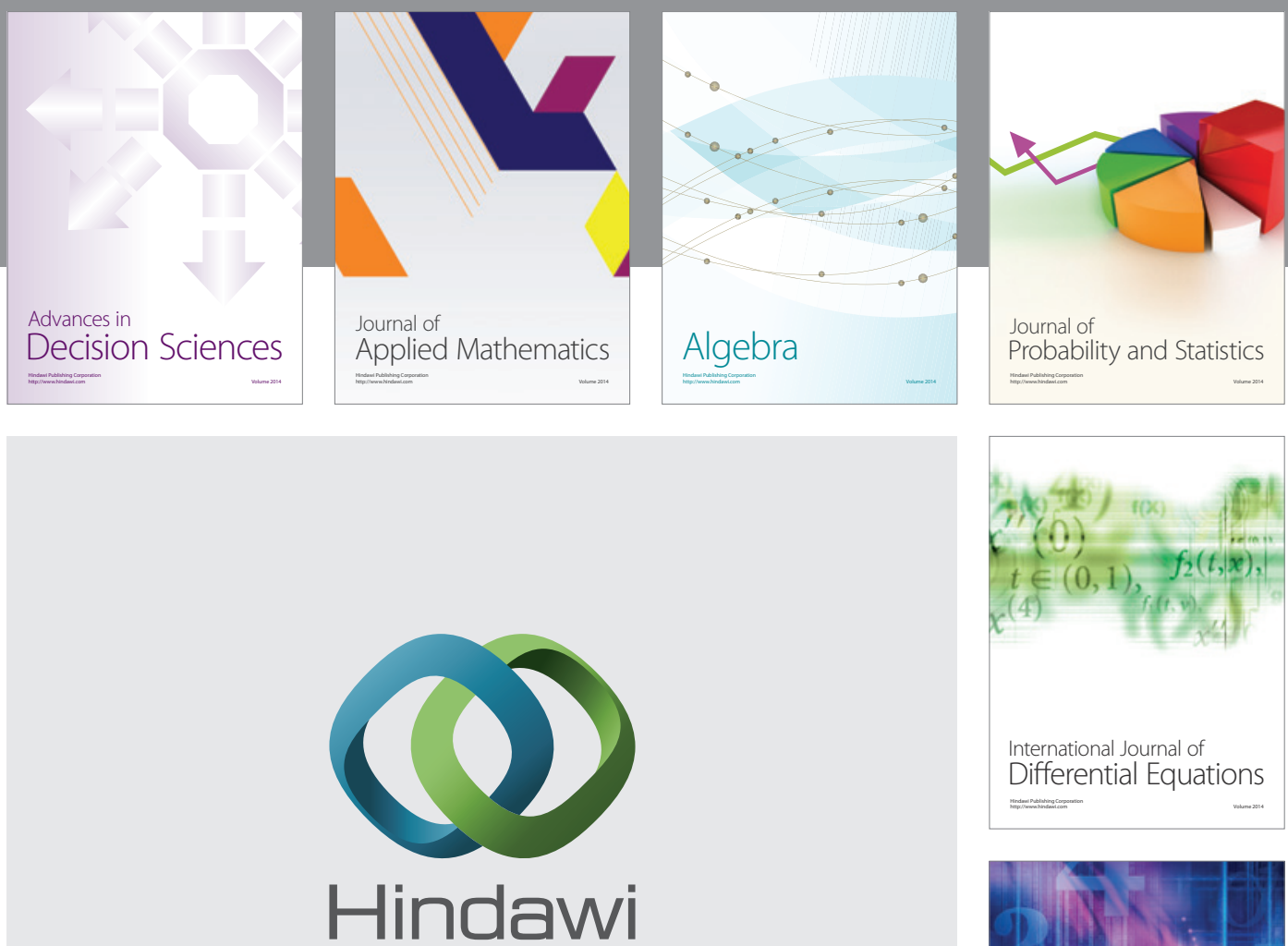

Submit your manuscripts at http://www.hindawi.com
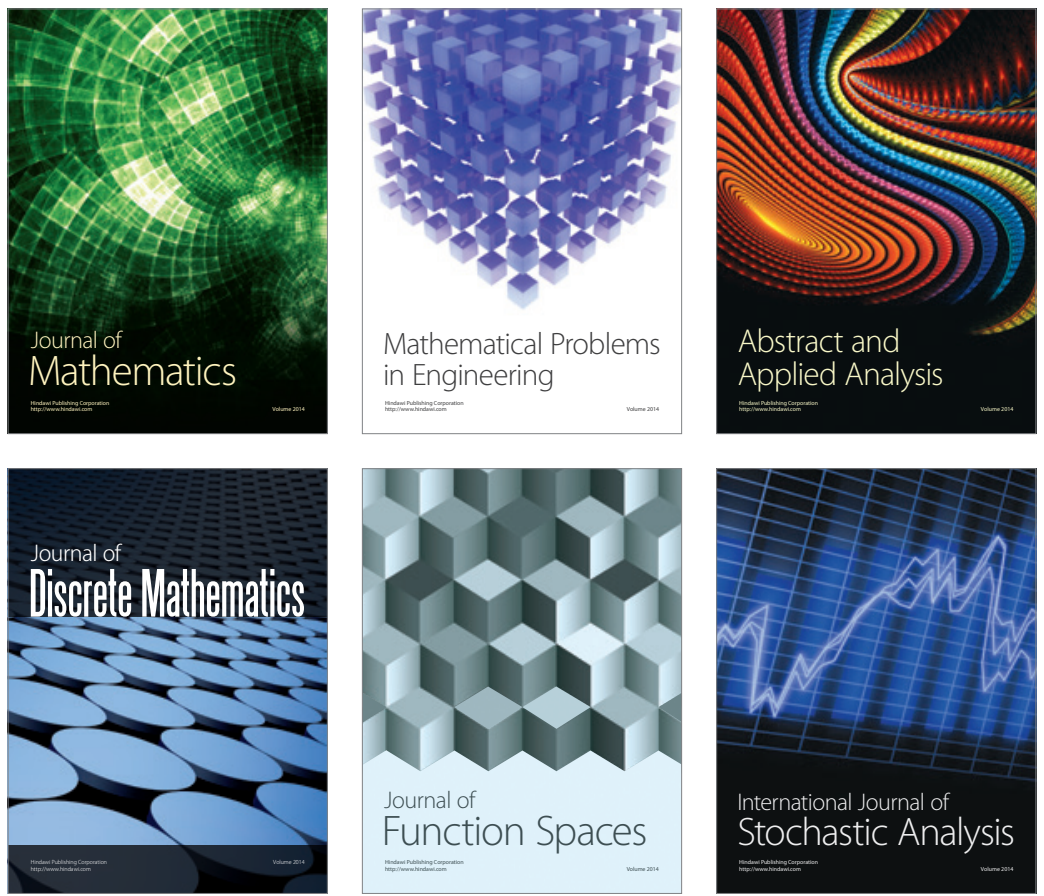

Journal of

Function Spaces

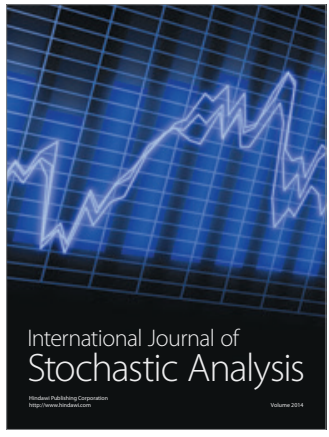

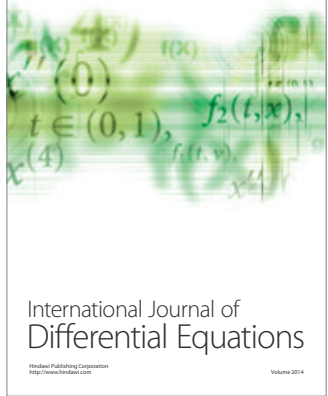
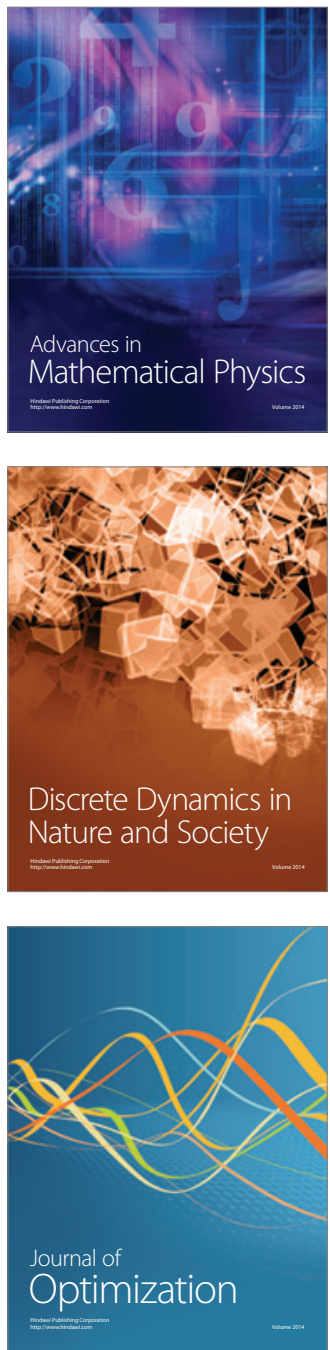